\title{
Stratégies de localisation des médecins généralistes français : mécanismes économiques ou hédonistes?
}

\author{
Eric Delattre * et Anne-Laure Samson **
}

En France, la densité médicale est élevée mais les médecins généralistes sont très inégalement répartis sur le territoire. Parce qu'elle renvoie à des questions d'équité et d'efficacité, cette mauvaise répartition constitue aujourd'hui un enjeu majeur de la régulation de la démographie médicale. Un échantillon exhaustif de 9000 médecins généralistes ayant débuté leur carrière libérale entre 1997 et 2002 est mobilisé afin d'analyser les déterminants des choix individuels de localisation des médecins généralistes et, ainsi, de rendre compte des outils qui pourraient être utilisés spécifiquement au niveau régional pour modifier leurs comportements de localisation.

Nous modélisons deux décisions : le choix du changement de région entre la date de la soutenance de la thèse et l'installation et le choix de la région d'exercice pour les seuls médecins qui quittent la région où ils ont soutenu leur thèse. Au travers de ces choix individuels, nous étudions en particulier l'attractivité monétaire des régions afin de déterminer dans quelle mesure les comportements des médecins pourraient être influencés par l'instauration de primes à l'installation dans les zones sous-dotées en médecins.

Nous observons un comportement marqué de sédentarité chez les médecins, qui s'installent en grande majorité dans la région de leur thèse. Les politiques doivent donc être orientées, en priorité, vers les étudiants en médecine. Nous montrons par ailleurs que les politiques d'incitations financières à l'installation dans des zones sous-dotées en médecins seraient susceptibles d'influencer la localisation des médecins, même si la modification de la répartition qui en résulterait serait quantitativement d'une faible ampleur. En effet, les primes devraient être d'un montant très élevé pour compenser la perte de qualité de vie consentie par les médecins. Les mécanismes financiers incitatifs ne peuvent donc pas être les seuls leviers de correction des inégalités de répartition des médecins.

\footnotetext{
* CREST (Ensai-UEB) et THEMA (UMR CNRS 8184)

${ }^{*}$ PSL, Université Paris Dauphine, LEDA-Legos

Nous tenons à remercier, pour l'ensemble de leurs remarques et suggestions, les deux rapporteurs anonymes de la revue ainsi que Muriel Barlet, Alain Carpentier, Arnold Chassagnon, Hugh Gravelle et Morgane Laouenan. Nous remercions également les participants des $10^{\text {es }}$ Journées LAGV (Marseille, juin 2010), du groupe de travail en Économie de la Santé (PSE, octobre 2010), des 32es Journées des Économistes de la Santé Français (Lyon, décembre 2010), du $2^{\text {nd }}$ Irdes Worskshop on Applied Health Economics and Policy Evaluation (Paris, juin 2011) et des séminaires de recherche de I'INRA-Rennes (février 2011) et du Health Economics Research Unit de l'université d'Aberdeen, Ecosse (mai 2011) pour leurs commentaires sur une première version de cet article. Ces travaux ont été réalisés avec le soutien de la Chaire Santé, placée sous l'égide de la Fondation Du Risque (FDR) en partenariat avec PSL, Université Paris-Dauphine, l'Ensae et la MGEN.
} 
$\mathbf{E}$ n France, la densité médicale est élevée comparativement aux autres pays de l'OCDE (OCDE, 2009), mais les généralistes libéraux sont très inégalement répartis sur le territoire (cf. carte 1). Parce qu'elle renvoie à des questions d'équité et d'efficacité, cette inégale répartition est aujourd'hui un enjeu majeur de la régulation de la démographie médicale.

En effet, du côté de la demande de soins, la mauvaise répartition des médecins entraîne des inégalités dans l'accès aux soins ainsi qu'un rationnement des patients. Selon la liste établie par les Missions régionales de santé (MRS), $4 \%$ de la population française vivrait dans une commune déficitaire ${ }^{1}$, avec de fortes disparités entre régions puisque ce chiffre atteindrait $10 \%$ pour les régions Bourgogne, Franche-Comté ou Champagne-Ardenne (Cnom, 2008).
En outre, du côté de l'offre de soins, des comportements de demande induite ont pu être identifiés pour les médecins généralistes du secteur 1 : lorsque la densité dans leur zone d'exercice augmente, les médecins compensent les rationnements qu'ils subissent sur le nombre de leurs patients en augmentant le volume de soins qu'ils délivrent au cours de chaque consultation. Ces comportements sont plus marqués dans les départements où la densité est élevée (Delattre et Dormont, 2003, 2005).

1. Une commune déficitaire est une commune où la densite de généralistes est inférieure d'au moins $30 \%$ à la moyenne nationale et le niveau d'activité des professionnels de santé est supérieur d'au moins $30 \%$ à la moyenne nationale (Cour des Comptes, 2007).

\section{Carte 1}

Répartition régionale des médecins généralistes libéraux (en 2007)
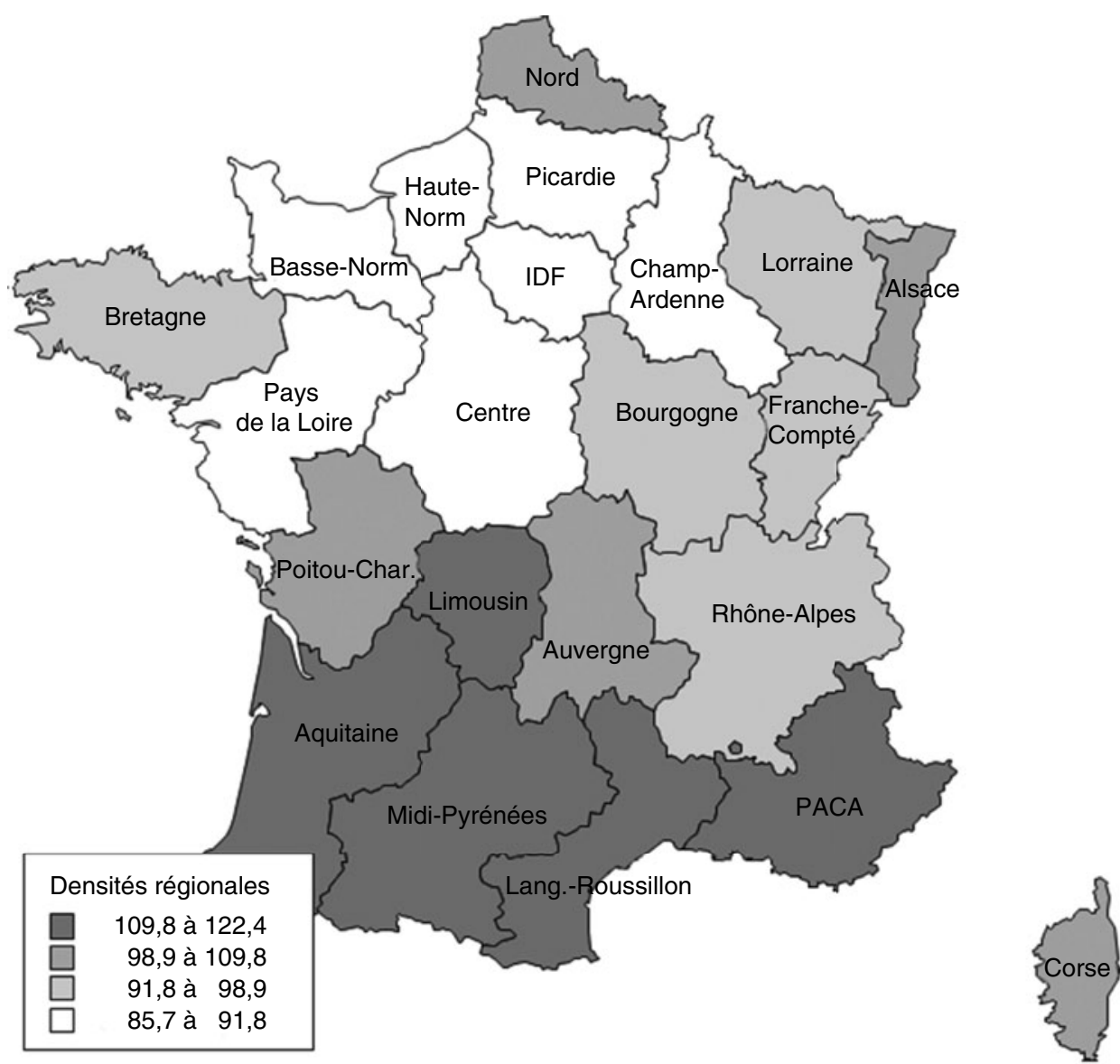

Lecture : plus la couleur de la région est foncée, plus la densité de médecins généralistes y exerçant est élevée. Ainsi, la densité la plus forte est observée dans les régions Limousin, Aquitaine, Midi-Pyrénées, Languedoc-Roussillon et PACA, avec des niveaux compris entre 109.8 et 122.4 généralistes pour 100000 habitants. Au niveau national, la densité moyenne est de 99 médecins généralistes pour 100000 habitants.

Champ : médecins généralistes libéraux exerçant en France métropolitaine en 2007.

Source : Eco-Santé 2010 (données DREES). 
Ces constats sont d'autant plus préoccupants qu'aujourd'hui la population des médecins vieillit, se féminise (et les femmes travaillent en moyenne $34 \%$ de moins que leurs confrères masculins; Dormont et Samson, 2009) et l'exercice de la médecine en libéral est de moins en moins attractif. En 2010, seulement $9,4 \%$ des médecins nouvellement inscrits au tableau de l'Ordre des médecins ont choisi un mode d'exercice libéral ; $70 \%$ ont choisi le salariat (Cnom 2011). Les conséquences de cette inégale répartition pourraient donc, à terme, s'aggraver.

À partir d'un échantillon exhaustif de médecins généralistes débutants, cette étude analyse les déterminants des choix de localisation des médecins au moment de leur installation. Elle vise à alimenter le débat sur les politiques susceptibles d'influencer les comportements de localisation des médecins et de contribuer, à terme, à améliorer leur répartition géographique.

\section{Comment améliorer la répartition géographique des médecins sur le territoire national ?}

La mauvaise répartition des médecins est une conséquence directe de leur liberté d'installation. À la fin de leurs études, les médecins choisissent librement de s'installer dans la région de leur choix. Rien ne les empêche de s'installer dans des zones déjà fortement dotées en médecins. Alors que des contraintes sur l'installation dans les zones considérées comme en surplus sont déjà en vigueur en Allemagne, au Royaume-Uni ou en Autriche (Bourgueil et al., $2007 b$ ), la régulation de la localisation géographique des médecins en France est limitée à deux types de mesures, plus incitatives que coercitives.

La première concerne la répartition géographique des étudiants en médecine. Chaque année depuis 1971, les variations du numerus clausus sont utilisées à des fins de rééquilibrage régional du nombre de médecins formés. Ainsi, entre 2000 et 2007, le numerus clausus est passé de 3850 à 7100 places, soit une hausse de près de $85 \%$ du nombre d'étudiants. Sur cette même période, l'augmentation du numerus clausus a été inférieure dans les régions très densément peuplées en médecins $(+77 \%$ et $+71 \%$ pour les régions Provence-Alpes-Côte d'Azur - PACA - et Aquitaine) et bien supérieure dans les régions de plus faible densité $(+102 \%$ et $+93 \%$ pour le Centre et la Picardie). Toutefois, la ventilation à la hausse du numerus clausus au niveau régional est nécessairement limitée par les disparités régionales en matière de capacités de formation (Vilain et Niel, 1999). En outre, étant donnée la longueur des études de médecine, les répercutions d'une telle politique ne sont visibles qu'à long terme. Enfin, rien ne garantit que les médecins formés dans une région s'y installeront ensuite pour exercer.

La seconde politique est plus récente. Depuis 2004, des aides financières sont accordées par l'État, l'Assurance maladie et/ou les collectivités locales afin d'inciter les nouveaux médecins à s'installer dans les zones sous dotées en médecins, considérées comme prioritaires par les MRS (Bourgueil et al., 2007a). Mais ces aides sont encore récentes, la plupart d'entre elles ne sont pas entrées en vigueur; elles n'ont donc encore concerné qu'un très faible nombre de médecins (Cnom, 2008).

\section{Comment expliquer la répartition des médecins sur le territoire?}

L'inégale répartition des médecins sur le territoire n'étant pas une spécificité française, il existe une littérature abondante qui vise à quantifier ces disparités géographiques, au moyen d'indicateurs de Gini notamment (voir par exemple Horev et al., 2004, pour les ÉtatsUnis ; Gravelle et Sutton, 2001, pour la GrandeBretagne ou Correia et Veiga, 2009, pour le Portugal). En revanche, très peu d'études s'interrogent sur les déterminants des choix de localisation des médecins. Les mesures prises par les différents pays de l'OCDE (Simoens et Hurst, 2006) semblent avoir été adoptées sans connaissance, a priori, de celles qui étaient susceptibles d'influencer le plus fortement les décisions de localisation des médecins.

Les quelques études françaises qui analysent les choix d'installation des médecins mettent en évidence l'impact des critères d'ordre personnel et/ou celui de facteurs macroéconomiques pour expliquer leurs comportements.

Ainsi, Bui et Lévy (2000) ont montré que $81 \%$ des jeunes médecins généralistes citent en premier lieu des critères d'ordre personnel (tels que la proximité de la famille, l'activité professionnelle du conjoint, l'agrément du cadre de vie) pour expliquer le choix de leur lieu d'installation. Le marché potentiel et les possibilités de rachat de cabinet jouent par ailleurs une part importante dans le processus de choix de la région. 
À partir d'une revue de la littérature, Bilodeau et Leduc (2003) montrent également que ce sont les facteurs personnels (connaissance antérieure de la région, lieu des études, etc.) qui conditionnent le plus le choix du lieu de pratique. Les facteurs contextuels tels que la réputation des établissements scolaires ont également un impact, mais ce n'est pas le cas de facteurs tels que le revenu moyen de la population ou de son niveau d'emploi.

Enfin, Couffinhal et al. (2002), à partir d'une analyse statistique menée au niveau cantonal dans laquelle ils ne disposent pas d'information d'ordre personnel, établissent que les facteurs explicatifs du choix d'installation des médecins ne sont pas tant le revenu et le niveau d'activité espéré que le contexte économique (niveau d'activité économique, revenus des habitants, etc.), démographique et les conditions de vie (présence d'un bureau de poste, de cinémas, etc.), dans le canton.

Nos données, administratives, ne fournissent aucune information sur les caractéristiques familiales, personnelles des médecins (présence d'un conjoint, situation professionnelle du conjoint, âge et nombre d'enfants, etc.). En conséquence, à la différence des études précédemment citées, notre analyse vise à quantifier l'importance d'une grande diversité de facteurs plus « macroéconomiques » sur les stratégies de localisation des médecins : l'attractivité monétaire des régions (revenu espéré), le dynamisme économique (revenu des habitants, taux de chômage, etc.), le dynamisme démographique et enfin des facteurs hédonistes (la qualité de vie, mesurée par différents indicateurs). Ce type d'approche a déjà été utilisé par Dionne et al. (1987) et plus récemment par Bolduc et al. $(1996,1997)$ qui étudient les choix de localisation des médecins québécois ou par Goddard et al. (2010) qui analysent les déterminants de la densité de généralistes dans les différentes régions anglaises. Tous mettent en évidence une influence forte de l'attractivité d'une région (mesurée par exemple par le revenu espéré) ou des variables hédoniques telles que le nombre de restaurants étoilés, la qualité de l'air, etc.) sur le choix de celle-ci par les médecins.

\section{Une analyse représentative des comportements individuels de localisation des médecins libéraux}

\section{Un échantillon exhaustif de médecins généralistes débutants}

Pour étudier les choix de localisation des médecins au moment de leur installation en libéral, nous utilisons un panel exhaustif de médecins généralistes fourni par la Caisse nationale de l'assurance maladie des travailleurs salariés (CNAMTS) qui contient des informations sur environ 9000 médecins généralistes ayant débuté leur activité entre 1997 et 2002 (cf. encadré 1). Le choix de localisation des médecins au moment de leur installation est défini par le choix de la région d'exercice. Ce niveau d'analyse est très agrégé : il existe probablement de fortes disparités intra-régionales en termes de comportement d'installation des médecins,

Encadré 1

\section{LES DONNÉES : UN ÉCHANTILLON EXHAUSTIF DE MÉDECINS GÉNÉRALISTES DÉBUTANTS}

\section{Base de données initiale}

Cette étude exploite les données d'un panel d'omnipraticiens libéraux ayant débuté leur activité entre 1997 et 2002. Le terme d'omnipraticiens regroupe ici les médecins généralistes ainsi que les médecins généralistes possédant un «mode d'exercice particulier » (MEP) comme l'acupuncture, l'homéopathie, la médecine du sport, etc. Ces derniers représentent environ $10 \%$ des omnipraticiens.

Ce panel est fourni par la Caisse d'assurance maladie des travailleurs salariés (CNAMTS). II reprend les statistiques relevées en date de remboursement par le Système national inter-régime (SNIR).
II s'agit d'un panel exhaustif : tous les omnipraticiens qui ont débuté leur activité libérale entre 1997 et 2002 sont présents dans le panel. Ce panel est non cylindré : les médecins qui se sont installés en 1997 sont observés sur une durée maximale de six années, ceux qui se sont installés en 1998 ne sont observés que sur une durée maximale de cinq ans, etc.

Au total, l'échantillon comporte 32000 observations relatives à environ 8200 médecins observés sur une durée maximale de six années.

Puisque nous étudions les choix de localisation au moment de l'installation, nous n'exploitons pas la dimension panel des données et nous nous concentrons 
certains départements ou communes étant plus attractifs que d'autres (les cantons ruraux sont généralement moins prisés que les grandes villes et les cantons urbains (Couffinhal et al., 2002)). Toutefois, une décomposition de la variance montre que $59 \%$ de la variance totale des niveaux

Encadré 1 (suite)

sur les comportements observés lors de la première année d'exercice. En conséquence, nous n'analysons pas les éventuels comportements de mobilité après la première installation des médecins. Dans le cas des médecins généralistes, les comportements de mobilité sont cependant rares. À partir d'une seconde source de données, un panel représentatif regroupant environ $10 \%$ des omnipraticiens en activité entre 1979 et 2004 , on observe que seuls $3 \%$ des généralistes ont changé de région au cours de leur carrière et $4 \%$ ont changé de type de commune d'exercice (ils sont passés d'un exercice en zone rurale à un exercice en zone urbaine ou vice versa). Se concentrer sur les choix de localisation au moment de l'installation donne une bonne indication de la localisation des médecins sur l'ensemble de leur carrière.

Pour chaque individu, nous connaissons son âge, son sexe, l'année et la région de la soutenance de sa thèse, l'année du début de son activité, le montant de ses honoraires, le niveau de son activité (décomposée en consultations, visites, actes de chirurgie et actes de radiologie). Nous savons également s'il s'agit d'un médecin «MEP » et son mode d'exercice (exercice libéral exclusif ou à temps partiel). Concernant la localisation géographique, nous connaissons la région, le département d'exercice ainsi que le type de commune dans laquelle le médecin exerce (commune rurale, petite, moyenne ou grande ville).

\section{Champ retenu}

Nous ne conservons que les médecins du secteur 1. Les médecins libéraux peuvent appartenir à deux secteurs de conventionnement : dans le secteur 1 , les tarifs sont fixés par des conventions nationales alors que dans le secteur 2 , les dépassements d'honoraires sont autorisés. Les médecins n'ont pu s'installer en secteur 2 qu'entre 1980 et 1992. Après 1992, son accès a été restreint aux seuls anciens chefs de clinique ou assistants des hôpitaux. De ce fait, le nombre de généralistes s'installant en secteur 2 entre 1997 et 2002 est marginal (moins de $2 \%$ chaque année) et ils ont des caractéristiques en termes d'activité et de localisation très différentes des autres médecins.

Par ailleurs, 310 médecins (3,3\% des médecins débutants) sont observés dans l'échantillon deux à trois années seulement après l'année de leur première installation en libéral. II s'agit probablement d'une erreur dans la collecte des données: nous n'observons pas leurs premières années de carrière. La localisation géographique actuelle mentionnée dans les données peut ne pas correspondre à leur localisation au moment de leur installation, celle que nous cherchons à expliquer. Nous décidons de les exclure de l'échantillon.

Enfin, pour des raisons de fiabilité de l'information, les omnipraticiens exerçant dans les Dom sont également exclus.

Notre échantillon final contient 7567 médecins débutants. En seulement six années, on observe deux modifications majeures de leurs caractéristiques (cf. tableau). D'une part, une importante féminisation de la profession : les femmes représentent $39,2 \%$ des médecins généralistes débutant en 1997 mais 44,6\% en 2002 ; par ailleurs, une hausse de l'âge moyen à l'installation. Celui-ci a augmenté de près de deux années sur la période, passant de 34,8 ans en 1997 à 36,7 ans en 2002. Cette hausse s'explique par l'augmentation de la durée des remplacements entre la soutenance de la thèse et l'installation en libéral. En revanche, l'âge à la thèse est resté très stable. En effet, sur la période, il n'y a eu aucune réforme modifiant les études de médecine et notamment leur durée.

Tableau

Principales caractéristiques des médecins débutants de l'échantillon

\begin{tabular}{|c|c|c|c|c|c|c|c|c|c|c|}
\hline \multirow{2}{*}{ Année } & \multirow{2}{*}{$\begin{array}{c}\text { Nombre } \\
\text { d'observations }\end{array}$} & \multirow{2}{*}{$\begin{array}{c}\% \text { de } \\
\text { femmes }\end{array}$} & \multicolumn{4}{|c|}{ Âge à l'installation } & \multicolumn{2}{|c|}{$\begin{array}{c}\text { Durée } \\
\text { des remplacements } \\
\text { (années) }\end{array}$} & \multicolumn{2}{|c|}{$\begin{array}{l}\text { Âge à la } \\
\text { thèse }\end{array}$} \\
\hline & & & $\begin{array}{c}1^{\mathrm{er}} \\
\text { quartile }\end{array}$ & médiane & moyenne & $\begin{array}{c}3^{e} \\
\text { quartile }\end{array}$ & médiane & moyenne & médiane & moyenne \\
\hline 1997 & 1489 & 39,2 & 31 & 33 & 34,8 & 37 & 2 & 4,0 & 30 & 30,8 \\
\hline 1998 & 1427 & 41,6 & 31 & 34 & 35,2 & 38 & 2 & 4,6 & 30 & 30,6 \\
\hline 1999 & 1245 & 40,4 & 31 & 34 & 35,4 & 38 & 2 & 4,8 & 30 & 30,6 \\
\hline 2000 & 1271 & 41,6 & 31 & 34 & 35,8 & 39 & 2 & 5,1 & 30 & 30,6 \\
\hline 2001 & 1118 & 44,4 & 31 & 34 & 36,1 & 39 & 2 & 5,2 & 30 & 30,9 \\
\hline 2002 & 1017 & 44,6 & 32 & 34 & 36,7 & 41 & 3 & 5,8 & 30 & 31,0 \\
\hline
\end{tabular}

Lecture : 39,2 \% des généralistes installés en 1997 sont des femmes. 25 \% des médecins se sont installés avant l'âge de 31 ans, la moitié s'est installée avant l'âge de 33 ans et $25 \%$ après 37 ans.

Champ : médecins généralistes libéraux de secteur 1, installés pour la première fois en libéral entre 1997 et 2002.

Source : échantillon de médecins généralistes débutants (CNAMTS). 
de densité est due aux écarts entre régions : raisonner au niveau régional nous permet de comprendre déjà une large part des stratégies de localisation des médecins. En outre, étant donnée la création des Agences régionales de santé (depuis le $1^{\text {er }}$ avril 2010), qui ont pour objectif de rationnaliser l'offre de soins ambulatoires sur le territoire, notre analyse permet de rendre compte de la pertinence d'outils qui pourraient être utilisés au niveau infrarégional par ces agences.

\section{Des régions françaises aux caractéristiques très contrastées}

On observe un phénomène déjà mis en évidence dans Dormont et Samson (2009) : il existe une relation décroissante entre niveaux de densité et revenus (cf. tableau 1). Les régions où la densité médicale est la plus faible sont aussi les régions où les médecins généralistes ont les revenus les plus élevés. En effet, la densité donne une indication du niveau de compétition entre les médecins. Si la densité d'une région est élevée, les médecins sont plus nombreux à se partager un même nombre de patients. Leur activité, et donc leurs revenus, sont plus faibles. Ainsi, les médecins de la région Champagne-Ardenne, région faiblement peuplée en médecins généralistes, gagnent $36 \%$ de plus que les médecins de la région PACA, région la plus densément peuplée en médecins généralistes ${ }^{2}$. Indépendamment de

2. II s'agit ici d'un écart "brut ». Lorsque l'on tient compte des différences dans la structure de l'offre et de la demande de soins, ces écarts, bien qu'amoindris, sont maintenus (Dormont et Samson, 2009) : l'écart de revenus entre la Picardie et PACA est de $28 \%$ ou de $21 \%$ entre Champagne-Ardenne et PACA.

Tableau 1

Principales caractéristiques des régions françaises (en 2007), classées par ordre croissant de densité de médecins généralistes

\begin{tabular}{|c|c|c|c|c|c|c|c|c|}
\hline & $\begin{array}{c}\text { Densité } \\
\text { de MG (pour } \\
100000 \text { hab.) }\end{array}$ & $\begin{array}{c}\text { Revenu } \\
\text { annuel } \\
\text { des MG } \\
(€)\end{array}$ & $\begin{array}{l}\text { Activité } \\
\text { annuelle } \\
\text { des MG } \\
\text { (nombre } \\
\text { d'actes) }\end{array}$ & $\begin{array}{c}\text { Conso } \\
\text { soins / } \\
\text { hab. } \\
(€)\end{array}$ & $\begin{array}{c}\text { Part des } \\
\text { hab. de + } \\
\text { de } 65 \text { ans } \\
(\%)\end{array}$ & $\begin{array}{c}\text { Part des } \\
\text { femmes } \\
\text { MG } \\
(\%)\end{array}$ & $\begin{array}{c}\text { Âge } \\
\text { moyen } \\
\text { des MG }\end{array}$ & $\begin{array}{l}\text { Heures } \\
\text { de soleil }\end{array}$ \\
\hline Centre & 85,8 & 66899 & 5513 & 229 & 18,3 & 19 & 47,1 & 1718 \\
\hline Île-de-France & 86,8 & 60744 & 4766 & 237 & 12,3 & 29 & 46,8 & 1581 \\
\hline Picardie & 88,2 & 78537 & 6503 & 233 & 14,8 & 19 & 46,4 & 1631 \\
\hline Basse-Normandie & 88,8 & 65749 & 5455 & 206 & 18,6 & 24 & 47,4 & 1651 \\
\hline Pays de la Loire & 91,0 & 64694 & 5489 & 213 & 16,8 & 25 & 46,8 & 1726 \\
\hline Haute-Normandie & 91,6 & 71267 & 6044 & 247 & 15,6 & 16 & 47,5 & 1608 \\
\hline Champagne-Ardenne & 91,8 & 76999 & 6471 & 226 & 16,6 & 23 & 48,1 & 1630 \\
\hline Bourgogne & 92,7 & 66184 & 5368 & 222 & 19,7 & 28 & 47,4 & 1706 \\
\hline Lorraine & 95,2 & 68692 & 5764 & 249 & 16,5 & 20 & 46,9 & 1699 \\
\hline Rhône-Alpes & 96,3 & 54942 & 4591 & 226 & 15,6 & 30 & 46,2 & 1987 \\
\hline Franche-Comté & 97,1 & 61304 & 5049 & 225 & 16,7 & 26 & 44,9 & 1765 \\
\hline Bretagne & 97,7 & 60186 & 5027 & 207 & 18,4 & 26 & 46,2 & 1695 \\
\hline Auvergne & 98,9 & 59378 & 5128 & 213 & 20 & 24 & 47,1 & 1849 \\
\hline Alsace & 103,1 & 66118 & 5429 & 274 & 15 & 15 & 46,6 & 1790 \\
\hline Poitou-Charentes & 103,5 & 66103 & 5478 & 217 & 20,3 & 22 & 47,0 & 1893 \\
\hline Nord-Pas-de-Calais & 103,7 & 75219 & 6323 & 274 & 14,3 & 17 & 46,7 & 1611 \\
\hline Corse & 103,9 & 56379 & 4169 & 281 & 19,9 & 13 & 47,2 & 2751 \\
\hline Midi-Pyrénées & 109,8 & 61630 & 5084 & 267 & 18,8 & 26 & 46,8 & 2012 \\
\hline Aquitaine & 114,2 & 61265 & 5083 & 264 & 19,2 & 24 & 47,3 & 1945 \\
\hline Limousin & 116,3 & 63516 & 5099 & 218 & 22,8 & 15 & 46,8 & 1873 \\
\hline Languedoc-Roussillon & 121,7 & 59910 & 4945 & 284 & 19,1 & 30 & 46,9 & 2510 \\
\hline Provence-Alpes-Côte d'Azur & 122,3 & 56314 & 4513 & 309 & 18,9 & 28 & 46,0 & 2881 \\
\hline Moyenne nationale & 99,0 & 63317 & 5246 & 244 & 16,3 & 24 & 46,7 & 2012 \\
\hline
\end{tabular}

Lecture : en moyenne dans la région Centre, la densité de médecins généralistes (MG) libéraux est de 85,8 médecins pour 100000 habitants. Le revenu annuel des médecins généralistes exerçant dans cette région est de $66899 €$, associé à un nombre annuel moyen de 5513 actes.

Champ : médecins généralistes libéraux exerçant en France métropolitaine en 2007.

Source : Eco-Santé 2010 et Météo-France. 
toute mesure financière incitative à l'installation dans des zones sous-dotées, ces statistiques montrent qu'il existe déjà un avantage financier à s'installer dans ces régions. Ce supplément de revenu s'accompagne toutefois d'un niveau d'activité plus élevé (colonne 3).

On observe également une relation positive entre le nombre d'heures de soleil par an des différentes régions, et la densité médicale. Ainsi, si la région Champagne-Ardenne a connu en moyenne 1630 heures de soleil en 2007, ce chiffre était de 2881 pour PACA. Si l'on voit dans l'ensoleillement une mesure indirecte de la qualité de vie, il existerait donc une relation décroissante entre qualité de vie et revenu moyen (cf. graphique). Il est probable que les médecins effectuent un arbitrage au moment de leur installation : ceux qui choisissent de s'installer dans des régions densément peuplées en médecins acceptent de percevoir des revenus plus faibles car ils bénéficient d'une meilleure qualité de vie. En revanche, les médecins qui choisissent de s'installer dans les régions peu densément peuplées en médecins perçoivent des revenus plus élevés pour compenser leur charge de travail accrue et leur moins bonne qualité de vie. Ce type de mécanisme n'est pas spécifique aux médecins. Ainsi, un des premiers travaux sur le sujet (Roback, 1982) explique les disparités régionales de niveaux de salaires moyens versés (entre 98 villes) par des caractéristiques géographiques. L'auteur montre qu'il existe une relation décroissante entre les salaires et des variables hédoniques telles que l'ensoleillement ou le nombre de jours sans usage de chauffage. Les résultats suggèrent que les individus acceptent des niveaux de salaires plus bas en contrepartie d'une qualité de vie supérieure.

Au-delà des différences dans la qualité de vie, l'inégale répartition des médecins sur le territoire pourrait s'expliquer par l'hétérogénéité de la demande de soins : les écarts de densité médicale entre régions refléteraient des différences d'état de santé et de besoins en santé de la population. La consommation de soins de santé par habitant en euros ainsi que la part des habitants de plus de 65 ans sont utilisés pour approximer la demande de soins dans les différentes régions (cf. tableau 1). Il existe des disparités interrégionales importantes et une corrélation positive entre consommation de soins et densité : lorsque la demande de soins est élevée,

\section{Graphique \\ Revenus moyens des médecins et nombre d'heures de soleil par région}

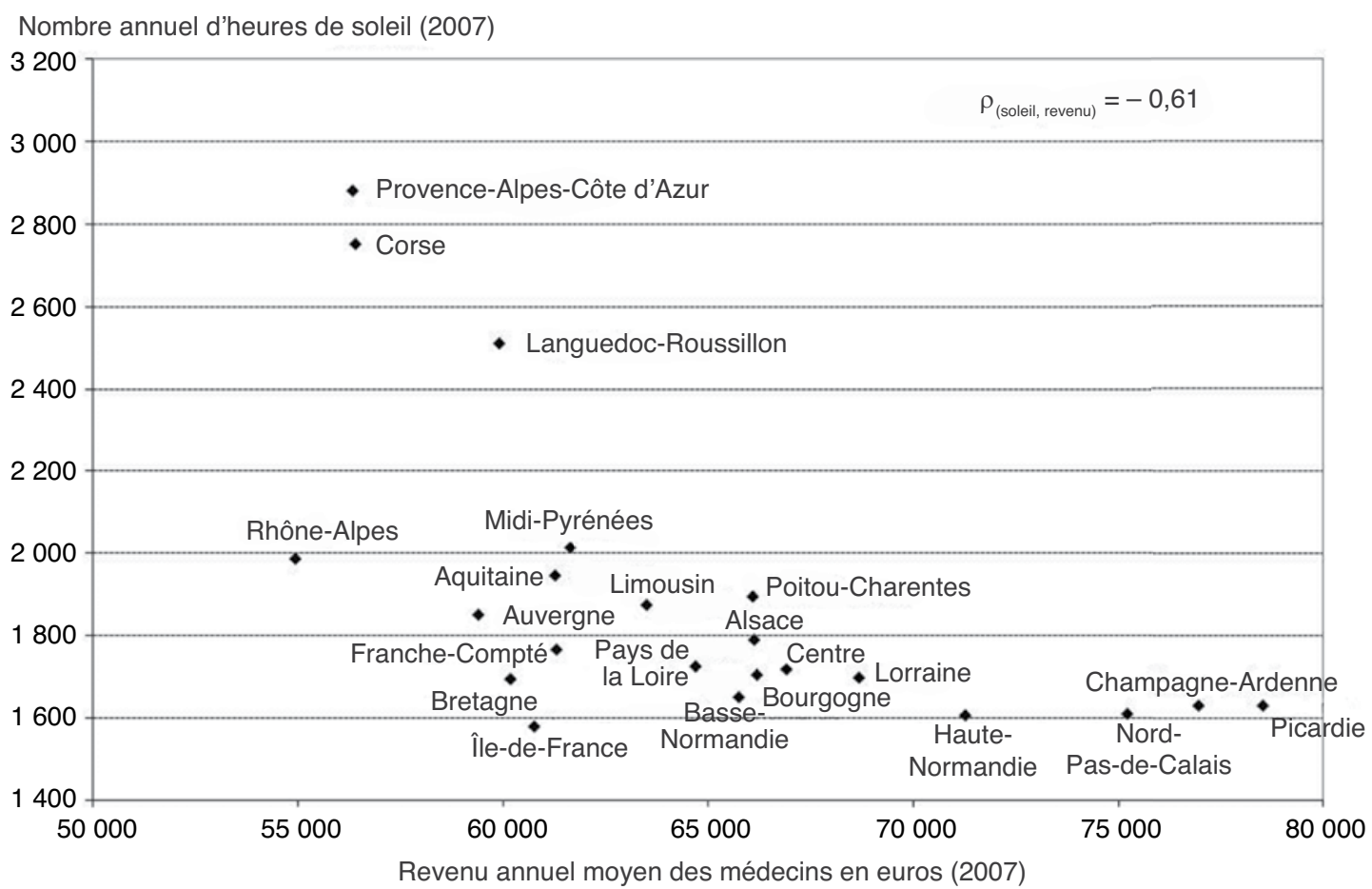

Lecture: en moyenne en 2007, les médecins généralistes exerçant dans la région Rhône-Alpes percevaient un revenu annuel de $54942 €$. Le nombre annuel d'heures de soleil dans cette région s'élevait à 1987 . Le coefficient de corrélation linéaire entre le nombre annuel d'heures de soleil et le revenu annuel moyen des médecins par région (noté $\rho_{\text {(soleil, revenu) }}$ ) est de - 0,61.

Champ : médecins généralistes libéraux exerçant en France métropolitaine en 2007.

Source : Eco-Santé 2010 et Météo-France. 
la densité l'est aussi. Toutefois, il est impossible de distinguer, dans cette corrélation, d'éventuels effets d'induction de la demande par les médecins. La part des plus de 65 ans apparait également plus élevée dans les régions du Sud de la France, ce qui peut aussi expliquer la plus forte consommation de soins dans ces régions. Cette analyse descriptive reste sommaire car elle est effectuée au niveau agrégé et elle ne contrôle pas des disparités dans la composition de la population par catégorie socioprofessionnelle (CSP), dans le taux de chômage, etc. Mais elle montre que l'offre de soins peut correspondre à une demande. Toutefois, les disparités interrégionales en matière de niveaux de densité ne correspondent pas exactement aux disparités en matière de demande de soins. Ainsi, certaines régions, où la part des habitants de plus de 65 ans est élevée (Bourgogne, Centre), se caractérisent par ailleurs par une faible densité médicale. La répartition des médecins sur le territoire dépend donc de facteurs autres que la seule demande de soins, qu'il importe de comprendre.

Les caractéristiques sociodémographiques des médecins (âge, sexe) sont proches entre les régions. Néanmoins, l'âge moyen des médecins est légèrement plus élevé dans les régions dans lesquelles la densité médicale est faible: les médecins exerçant dans la région Centre sont par exemple significativement plus âgés que ceux exerçant en PACA. Les besoins futurs en médecins seront donc probablement plus élevés dans les régions où la densité est faible, aggravant, à terme, les conséquences de la mauvaise répartition.

\section{Où les médecins débutants s'installent-ils ?}

La forte hétérogénéité spatiale dans l'attractivité des régions se retrouve lorsque l'on examine les comportements de localisation des médecins débutants.

$74 \%$ d'entre eux s'installent dans la région dans laquelle ils ont fait leurs études de médecine et soutenu leur thèse ${ }^{3}$. Certaines régions retiennent toutefois plus leurs étudiants et d'autres sont beaucoup plus exportatrices d'étudiants. L'analyse économétrique présentée ciaprès analyse les facteurs explicatifs de cette hétérogénéité entre les régions.

Les médecins sont peu mobiles, en comparaison de ce qu'on observe chez les étudiants issus de filières scientifiques dans l'enseignement supérieur. À titre d'exemple, pour $62 \%$ des diplômés des écoles d'ingénieurs en 2001 et $43 \%$ des diplômés d'un troisième cycle universitaire en mathématiques ou sciences, la région du premier emploi est différente de la région de formation (Cereq, 2003). L'âge moyen à l'installation des médecins (33 à 35 ans), bien supérieur à celui des futurs cadres (25 ans), peut expliquer une partie de cette différence, leurs coûts de mobilité étant plus élevés.

Pour les $25 \%$ de médecins qui quittent leur région de thèse afin de s'installer dans une autre région, il existe également de fortes disparités dans le choix des régions, certaines apparaissant beaucoup plus attractives que d'autres. L'analyse économétrique de la section suivante analyse par ailleurs les raisons de ces différences d'attractivité entre les régions.

\section{Analyse économétrique des choix de localisation des médecins généralistes}

nalyser les comportements de localisation
des médecins nous conduit à modéliser
deux décisions : i) celle de changer de région
entre la thèse et l'installation en libéral (équa-
tion 1 ) ; ii) celle du choix de la région d'exercice
pour les médecins qui choisissent de changer de
région à l'issue de leur thèse (équation 2$)^{4}$.

\section{Cadre d'analyse}

La première décision peut être étudiée simplement à l'aide d'un modèle Logit (équation 1) dans lequel on explique la probabilité de s'installer dans une autre région à l'issue de la thèse.

Pour analyser la seconde décision, celle du choix de la région d'exercice, nous nous inspirons de la méthodologie employée par Bolduc

\footnotetext{
3. Ce constat est un phénomène déjà observé à partir d'autres sources de données : Vilain et Niel (1999) le quantifient à $69 \%$ et Bessière et al. (2004) à $73 \%$. Ces chiffres seront probablement amenés à évoluer vers une plus grande mobilité des étudiants avec la réforme des études médicales de 2004 : l'introduction des épreuves classantes nationales offre désormais la possibilité aux étudiants d'effectuer leur troisième cycle de médecine dans une autre région que celle dans laquelle ils ont débuté leurs études de médecine.

4. Une analyse plus complète consisterait à modéliser simultanément les deux décisions, en tenant compte de l'endogénéite de la décision de changer de région à l'issue de la thèse. Ce type de modélisation est cependant complexe à mettre en œuvre dans le cas d'un modèle Logit conditionnel. Dans la $2^{\text {ème }}$ équation, estimée à partir d'un sous échantillon de médecins mobiles après leur thèse, nous adoptons une approche plus descriptive, séquentielle et qui ne tient pas compte de la potentielle endogénéité du changement de région.
} 
et al. (1996) qui étudient les choix de localisation des médecins généralistes québécois.

Nous supposons que chaque médecin choisit de s'installer dans la région qui lui procure la plus grande utilité. Très schématiquement, l'utilité d'un médecin à exercer dans une région dépend de ses caractéristiques individuelles, du niveau de sa consommation, du nombre d'heures de loisir et des caractéristiques de la région. Chaque médecin maximise son utilité pour déterminer son lieu d'activité, sous la contrainte de la demande potentielle qui s'adresse à lui et qui détermine son revenu.

La demande qui s'adresse à chaque médecin dépend de l'état de santé et des caractéristiques sociodémographiques de la population ainsi que du nombre de médecins qui exercent dans la région (si la densité médicale d'une région est élevée, le niveau de la demande potentielle qui s'adresse à chaque médecin est plus faible).

Notre approche consiste à utiliser des méthodes économétriques de choix discrets et à estimer un modèle Logit conditionnel (équation 2), dans lequel les différentes alternatives correspondent aux 22 régions potentielles dans lesquelles le médecin peut s'installer (cf. annexe).

\section{Les facteurs explicatifs des comportements individuels de localisation}

Les deux équations utilisent les mêmes variables explicatives :

- des variables spécifiques aux médecins : le sexe et l'âge à l'installation ;

- des effets fixes régionaux. Ces constantes captent l'influence des caractéristiques inobservées (ou non) mais constantes dans le temps de chaque région (région de thèse ou région d'installation) et reflètent leur attractivité, toutes choses égales par ailleurs ${ }^{5}$.

5. Pour des raisons d'identification, une de ces constantes sert de région de référence et son coefficient est nul. Dans les deux spécifications, nous choisissons comme référence la région Picardie.

Encadré 2

\section{ENRICHISSEMENT DE LA BASE : LES VARIABLES EXPLICATIVES POTENTIELLES DES COMPORTEMENTS INDIVIDUELS}

Pour comprendre les comportements individuels de localisation des médecins, nous utilisons deux types de variables : i) des variables définies au niveau individuel (le médecin), qui permettent d'étudier l'influence des caractéristiques individuelles des médecins (âge, sexe notamment) sur le choix d'une région plutôt qu'une autre ; ii) des variables définies à un niveau plus agrégé (la région), afin de mettre en évidence quelles caractéristiques régionales (situation économique ou démographique, qualité de la vie) influencent le plus le choix des médecins pour une région. Le panel initial fournit des informations sur les caractéristiques individuelles des omnipraticiens. En revanche, il ne contient aucune information sur les caractéristiques de la région dans laquelle ils s'installent ou ont effectué leurs études. Plusieurs autres sources de données ont donc été mobilisées afin d'intégrer au panel initial des variables caractérisant la situation démographique, économique et environnementale de chacune des régions françaises. Nous pouvons les classer en trois catégories:

i) Les variables régionales relatives aux caractéristiques de la médecine ambulatoire :

L'activité moyenne (nombre moyen de consultations, visites, actes de radiologie et de chirurgie) et les honoraires moyens des médecins de chaque région sont obtenus à partir d'un second panel de médecins généralistes. À la différence de notre panel exhaustif de généralistes débutants, ce panel, également fourni par la CNAMTS, est un tirage au $10^{\mathrm{ème}}$ de l'ensemble des médecins généralistes en activité sur la période 1979-2004. II ne comporte donc pas uniquement les médecins débutants. En sélectionnant les médecins de plus de six années d'ancienneté (que nous supposons être le nombre d'années nécessaires à la constitution de leur clientèle, cf. Delattre et Dormont, 2005) et exerçant entre 1997 et 2002 , nous obtenons alors l'activité et les honoraires moyens annuels des médecins installés dans chaque région.

Les revenus moyens des médecins (c'est-à-dire les honoraires nets de l'ensemble des charges professionnelles) ont été obtenus, pour chaque région et chaque année entre 1997 et 2002, à partir des déclarations des bénéfices non commerciaux, répertoriées dans les statistiques fiscales de la Direction générale des finances publiques.

Enfin, la densité moyenne de médecins généralistes, la densité moyenne de médecins spécialistes et la densité 
- des variables spécifiques à la région, qui sont constantes ou non dans la dimension temporelle et qui caractérisent la région de thèse (équation 1) ou la région d'installation (équation 2); cf. encadré 2.

L'effet de la majorité de ces variables sur la probabilité de choisir une région est a priori connu, à l'exception de certaines. C'est le cas pour le revenu moyen espéré dans chaque région. D'un côté, les généralistes sont probablement plus à même de s'installer dans des régions dans lesquelles le revenu espéré est élevé. Toutefois, les médecins de secteur 1 étant payés à l'acte avec des tarifs fixes, un revenu moyen élevé dans une région est associé à un niveau d'activité élevé (cf. tableau 1). La probabilité pour un médecin de s'installer dans une telle région dépendra donc de sa préférence pour le « loisir ».

L'effet est également ambigu pour les trois variables de densité médicale : la densité de médecins généralistes (le nombre de médecins généralistes pour 100000 habitants), la densité de médecins spécialistes dans la région et la densité de médecins généralistes partis à la retraite en $\mathrm{t}-1$.

Inclure la densité de médecins spécialistes permet d'évaluer dans quelle mesure les médecins généralistes considèrent les spécialistes comme des «concurrents» potentiels lorsqu'ils choisissent une région : cette variable mesure le degré de complémentarité ou de substituabilité entre généralistes et spécialistes. Si c'est la complémentarité qui domine, une augmentation de la densité de spécialistes conduira à une augmentation de la demande qui s'adresse à chaque généraliste et ces derniers préféreront alors s'installer dans les régions densément peuplées en médecins spécialistes.

La densité de médecins généralistes partis à la retraite en t-1 permet d'approximer la part de marché disponible pour les médecins généralistes qui débutent leur activité dans une région à une année donnée. En effet, pour débuter leur carrière, les médecins peuvent racheter la clientèle d'un médecin qui part à la retraite ou la constituer eux-mêmes. Un nombre élevé de médecins partant à la retraite (rapporté à la population) signifie que les nouveaux médecins ont plus de possibilités de rachat de cabinet, une part de marché potentielle plus élevée et une compétition moindre en début de carrière ce qui peut favoriser la constitution de leur clientèle (Dormont et Samson, 2009) et les inciter à s'installer dans cette région.

Enfin, l'effet de la variable de densité de médecins généralistes est a priori de signe inconnu. D'un côté, la densité indique le degré de

Encadré 2 (suite)

moyenne de médecins généralistes partis à la retraite en $t-1$, par année et région ainsi que taux d'équipement pour personnes âgées (i.e. le nombre de lits en maisons de retraite, logements-foyers, unités de soins longue durée et résidences d'hébergement temporaire, pour 1000 personnes de 75 ans et plus) sont disponibles dans la base de données Eco-santé (chiffres Drees).

ii) Les variables relatives aux caractéristiques sociodémographiques et économiques de la région

Pour approximer l'état de santé de la population, on utilise la part des habitants de plus de 75 ans (source : EcoSanté, chiffres Insee) par année (1997-2002) et région. Pour donner une indication du développement économique de la région, on utilise le revenu moyen des habitants de la région et le taux de chômage (source : EcoSanté, chiffres Insee), également par année et région.

iii) Les « aménités » ou variables hédoniques

Plusieurs variables susceptibles de capturer l'impact des «aménités " (c'est-à-dire l'agrément apporté par le climat, la qualité de vie, les équipements) de chaque région sont envisagées : le nombre d'heures de soleil (année 2000 ; source: Météo France), le prix du $\mathrm{m}^{2}$ des appartements (années 1997-2002, source : Ifen, Institut français de l'environnement, Ministère de l'écologie, de l'énergie, du développement durable et de la mer), le nombre de chambres d'hôtels par habitant (recensement 1999, source : Statistiques locales de l'Insee), une indicatrice qui vaut 1 si la région comporte des communes littorales (recensement 1999, source: Ifen), la part de la population vivant dans des communes rurales (recensement 1999, source: Statistiques locales de I'Insee) et le nombre de Rotary Clubs (année 2009, source : site du Rotary Club).

Ces variables ont toutes été créées à deux niveaux : la région d'installation du médecin et sa région de thèse, afin de les utiliser comme variables explicatives dans l'équation (1) - la probabilité de changer de région après la thèse - et dans l'équation (2) - le choix de la région d'exercice. 
compétition entre généralistes. À qualité de vie et niveau moyen de revenu donnés, le niveau de la densité reflète alors la plus ou moins grande facilité des médecins à atteindre un certain niveau de revenu. Une forte densité peut donc désinciter les médecins à l'installation dans une région. D'un autre côté, la densité reflète également l'environnement social et professionnel auquel un médecin sera confronté s'il choisit de s'installer dans une région. Si la densité de généralistes est élevée, les médecins sont aussi moins isolés, ce qui peut faciliter leurs conditions d'exercice, et notamment leur ouvrir davantage de possibilités d'installation en cabinet de groupe, ce dernier permettant le partage des charges et une organisation plus souple du temps de travail (Baudier et al., 2010). Pour ces raisons, les médecins peuvent au contraire être incités à s'installer dans une région de plus forte densité.

Dans les deux spécifications, les variables définies au niveau régional ne peuvent être estimées simultanément avec les effets fixes régionaux que si elles ont suffisamment de variance temporelle. La plupart de nos variables hédoniques (nombre d'heures de soleil, nombre de chambres d'hôtel par habitants, etc.) sont, par construction, constantes; elles ne peuvent pas être estimées simultanément avec les constantes régionales. Pour d'autres indicateurs variant dans le temps, la part de la variance interrégionale dans la variance totale est trop élevée ( $98 \%$ pour la densité de médecins généralistes, $93 \%$ pour la part des habitants de plus de 75 ans) pour que ces variables soient inclues simultanément avec les constantes régionales. Seules quelques variables (le revenu moyen des médecins ou le revenu des habitants par exemple) ont une variabilité temporelle plus importante.

De ce fait, nous présentons, pour chacune des deux équations estimées, deux variantes qui diffèrent par la liste des variables explicatives introduites :

i) la première inclut les variables spécifiques au médecin ainsi que les effets fixes régionaux et les quelques variables explicatives définies au niveau régional qui ont suffisamment de variance temporelle ;

ii) la seconde inclut les variables spécifiques au médecin. Elle n'inclut pas les effets fixes régionaux, mais inclut l'ensemble des variables explicatives définies au niveau régional.
Dans la première variante, la valeur des effets fixes régionaux permet de quantifier l'ampleur des différences d'attractivité des régions. $\mathrm{La}$ seconde variante vise à expliquer ces écarts, en se concentrant sur l'information contenue dans les variables explicatives définies au niveau régional.

\section{Les régions ensoleillées et littorales retiennent plus leurs étudiants}

Dans la première équation, nous estimons la probabilité, pour un médecin, de quitter sa région de formation à l'issue de sa thèse (cf. carte 2 et tableau 2).

Puisque $75 \%$ des médecins s'installent dans la région de leur thèse, des politiques ciblées sur les étudiants en médecine pourraient être efficaces pour influencer ensuite la localisation géographique des médecins. Toutefois, il existe de fortes hétérogénéités entre les régions (cf. carte 2).

Dans les régions représentées en blanc, la probabilité relative de départ après la formation pour s'installer dans une autre région est significativement plus faible que dans la région de référence (Picardie). Ainsi, le Sud et l'Ouest de la France, et plus généralement l'ensemble des régions littorales, sont des régions qui retiennent fortement leurs étudiants, la plus faible probabilité relative de départ étant obtenue pour la région PACA. En revanche, la probabilité relative de quitter les régions les plus foncées (Île-de-France et Limousin) à l'issue de sa thèse pour s'installer dans une autre région est significativement supérieure à la probabilité observée en Picardie.

Les régions attractives pour les médecins sont très différentes de celles des futurs cadres issus de filières scientifiques de l'enseignement supérieur (Cereq, 2003). Ces futurs cadres sont moins nombreux à quitter l'Île-de-France, l'Alsace, la Lorraine ou la Picardie. Ils sont en revanche nombreux à quitter le Languedoc-Roussillon, la région Pays de la Loire ou l'Aquitaine, alors que ces régions sont, elles, très attractives pour les médecins. Les jeunes cadres recherchent probablement à s'installer avant tout dans des régions où le marché du travail est actif, centré sur les emplois les plus qualifiés et où ils peuvent espérer des revenus plus élevés, compte tenu de leurs qualifications. Mais comment expliquer ces différences d'attractivité pour les médecins? L'estimation de l'équation (1) sans les effets fixes régionaux, mais avec les 
différentes variables relatives à la région de thèse, permet de répondre à cette question ${ }^{6}$. Notons que l'Île-de-France est une région très particulière : il s'agit de la région qui forme le plus de médecins (près de $15 \%$ des médecins y ont été formés) mais c'est aussi la région la plus « exportatrice » d'étudiants (43\% des étudiants qui y ont été formés la quittent à l'issue de leur thèse, contre $26 \%$ en moyenne). Le poids de cette région peut donc fortement influencer les coefficients estimés. Nous présentons alors les résultats de l'estimation sans effets fixes régionaux sur l'ensemble des médecins (équation 1-b) et sur le sous-échantillon de médecins ayant soutenu leur thèse hors Îlede-France (équation 1-c). Nous commentons ci-dessous essentiellement les résultats de l'équation 1-b.
Les résultats des estimations confirment que les régions ensoleillées et littorales retiennent le plus leurs étudiants. Pour un médecin, la probabilité relative de quitter une région très fortement ensoleillée est $46 \%$ plus faible que s'il avait soutenu sa thèse dans une région moyennement ensoleillée. En outre, si les médecins ont fait leurs études dans une région littorale, la probabilité relative qu'ils quittent leur région de thèse diminue de $38 \%$.

6. La corrélation entre les prédictions obtenues à partir de ces deux estimations est de 0,91: les effets fixes régionaux synthétisent bien l'information contenue dans l'ensemble des variables explicatives définies au niveau régional. Ce constat est renforcé par le fait que les coefficients estimés des variables ayant suffisamment de variance temporelle varient très peu entre les deux types de spécifications.

\section{Carte 2}

Estimation de la probabilité de changer de région à l'issue de la thèse (équation 1)

Valeur des odd-ratios associés aux effets spécifiques régionaux

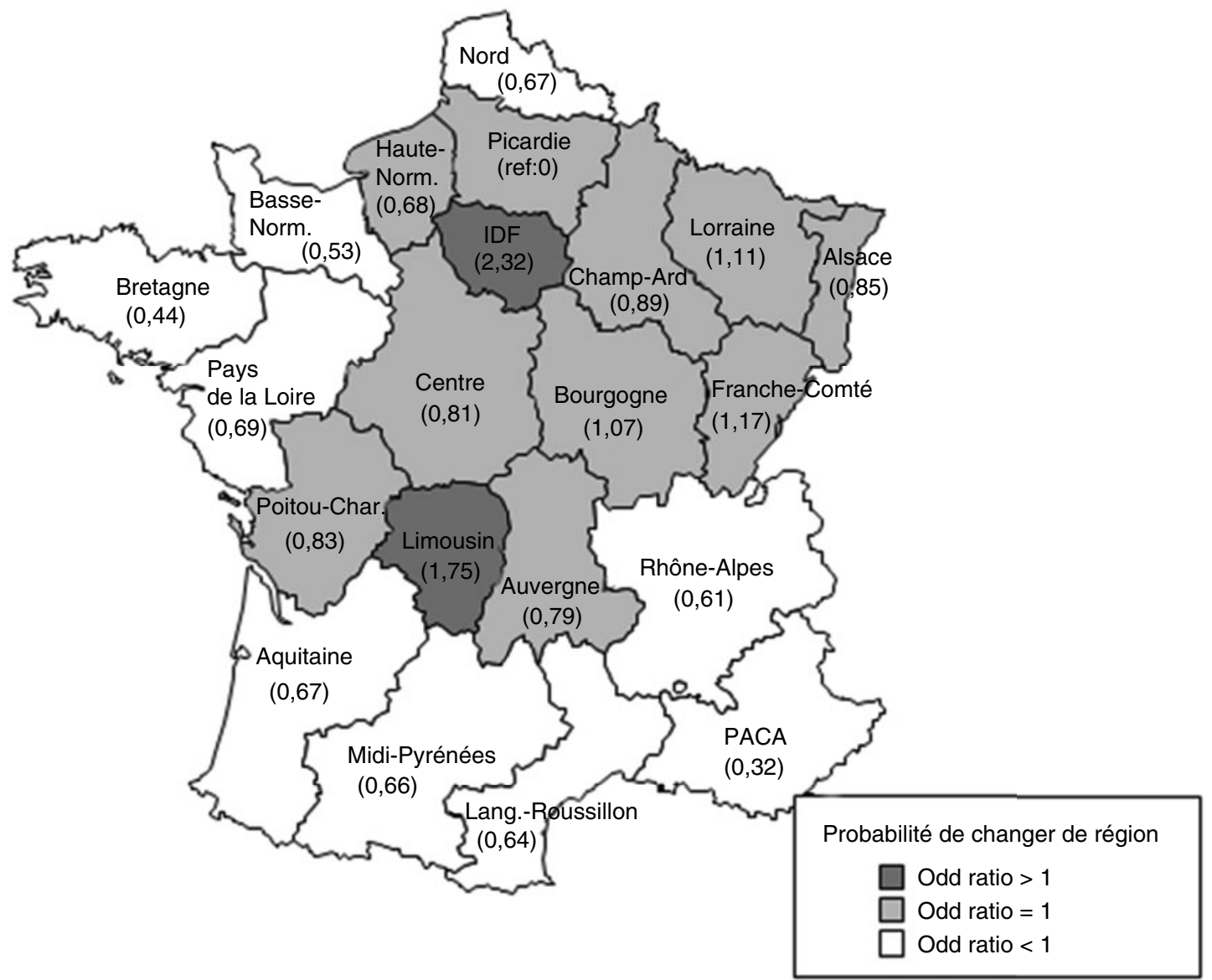

Lecture : la Corse ne figure pas sur ce graphique car il n'existe pas d'université de médecine dans cette région. La valeur des odd-ratios figure entre parenthèses sous le nom des régions. Un médecin formé en île-de-France a une probabilité relative de quitter sa région de formation 2,3 fois plus élevée qu'un médecin formé en Picardie (la région de référence).

Champ : médecins généralistes libéraux de secteur 1, installés pour la première fois en libéral entre 1997 et 2002.

Source : échantillon de médecins généralistes débutants (CNAMTS). 
Les médecins semblent par ailleurs sensibles au degré de compétition qui prévaut dans leur région de thèse : ils ont 1,49 fois plus de chances de quitter leur région de thèse lorsque

Tableau 2

Estimation de la probabilité de changer de région à l'issue de la thèse (équation 1)

Valeur des odd-ratios

\begin{tabular}{|c|c|c|c|}
\hline & $\begin{array}{c}\text { Équation (1-a) } \\
\text { (avec effets fixes régionaux) }\end{array}$ & $\begin{array}{c}\text { Équation (1-b) } \\
\text { (sans effets fixes régionaux) }\end{array}$ & $\begin{array}{c}\text { Équation (1-c) } \\
\text { (sans effets fixes régionaux } \\
\text { et sans l'lle de France) }\end{array}$ \\
\hline \multicolumn{4}{|l|}{ Variables caractéristiques du médecin } \\
\hline $\begin{array}{l}\text { Sexe } \\
\text { (Ref. : homme) }\end{array}$ & $\begin{array}{c}0,969 \\
(0,055)\end{array}$ & $\begin{array}{c}0,978 \\
(0,055)\end{array}$ & $\begin{array}{c}0,957 \\
(0,060)\end{array}$ \\
\hline $\begin{array}{l}\text { Thèse avant } 30 \text { ans } \\
\text { (Ref. : entre } 30 \text { et } 32 \text { ans) }\end{array}$ & $\begin{array}{l}1,348^{\star \star \star} \\
(0,089)\end{array}$ & $\begin{array}{l}1,356^{\star \star \star} \\
(0,089)\end{array}$ & $\begin{array}{l}1,417^{\star \star \star} \\
(0,103)\end{array}$ \\
\hline $\begin{array}{l}\text { Thèse après } 33 \text { ans } \\
\text { (Ref. : entre } 30 \text { et } 32 \text { ans) }\end{array}$ & $\begin{array}{l}0,695^{\star \star \star} \\
(0,053)\end{array}$ & $\begin{array}{l}0,702^{\star \star \star} \\
(0,053)\end{array}$ & $\begin{array}{c}0,763 \\
(0,065)\end{array}$ \\
\hline $\begin{array}{l}\text { Installation avant } 32 \text { ans } \\
\text { (Ref. : entre } 32 \text { et } 38 \text { ans) }\end{array}$ & $\begin{array}{l}0,520^{\star \star *} \\
(0,039)\end{array}$ & $\begin{array}{l}0,522^{\star \star \star} \\
(0,039)\end{array}$ & $\begin{array}{l}0,547^{\star \star \star} \\
(0,045)\end{array}$ \\
\hline $\begin{array}{l}\text { Installation après } 39 \text { ans } \\
\text { (Ref. : entre } 32 \text { et } 38 \text { ans) }\end{array}$ & $\begin{array}{l}2,132^{\star \star \star} \\
(0,147)\end{array}$ & $\begin{array}{l}2,119^{\star \star \star} \\
(0,145)\end{array}$ & $\begin{array}{l}2,434^{\star \star *} \\
(0,186)\end{array}$ \\
\hline $\begin{array}{l}\text { Année } 1998 \\
\text { (Ref. : année 1997) }\end{array}$ & $\begin{array}{c}0,891 \\
(0,097)\end{array}$ & $\begin{array}{c}0,882 \\
(0,088)\end{array}$ & $\begin{array}{c}0,829 \\
(0,093)\end{array}$ \\
\hline $\begin{array}{l}\text { Année } 1999 \\
\text { (Ref. : année 1997) }\end{array}$ & $\begin{array}{c}0,911 \\
(0,096)\end{array}$ & $\begin{array}{c}0,931 \\
(0,094)\end{array}$ & $\begin{array}{l}1,001 \\
(0,119)\end{array}$ \\
\hline $\begin{array}{l}\text { Année } 2000 \\
\text { (Ref. : année 1997) }\end{array}$ & $\begin{array}{l}0,857 \\
(0,102)\end{array}$ & $\begin{array}{c}0,892 \\
(0,102)\end{array}$ & $\begin{array}{c}0,995 \\
(0,136)\end{array}$ \\
\hline $\begin{array}{l}\text { Année } 2001 \\
\text { (Ref. : année 1997) }\end{array}$ & $\begin{array}{l}0,781 \\
(0,102)\end{array}$ & $\begin{array}{c}0,857 \\
(0,108)\end{array}$ & $\begin{array}{l}0,915 \\
(0,142)\end{array}$ \\
\hline $\begin{array}{l}\text { Année } 2002 \\
\text { (Ref. : année 1997) }\end{array}$ & $\begin{array}{c}0,704 \\
(0,112)\end{array}$ & $\begin{array}{l}0,786 \\
(0,114)\end{array}$ & $\begin{array}{c}0,907 \\
(0,159)\end{array}$ \\
\hline \multicolumn{4}{|l|}{ Variables caractéristiques de la région de thèse } \\
\hline Effets fixes régionaux & cf. carte 2 & - & - \\
\hline $\begin{array}{l}\text { Revenus Med Génér. < Q1 (= } 51700 €) \\
\text { (Ref. : Q1-Q3) }\end{array}$ & $\begin{array}{c}1,153 \\
(0,124)\end{array}$ & $\begin{array}{l}1,125 \\
(0,095)\end{array}$ & $\begin{array}{l}1,068 \\
(0,111)\end{array}$ \\
\hline $\begin{array}{l}\text { Revenus Med Génér. > Q3 (= } 60624 €) \\
\text { (Ref. : Q1-Q3) }\end{array}$ & $\begin{array}{l}1,305^{\star \star} \\
(0,173)\end{array}$ & $\begin{array}{l}1,392^{\star \star *} \\
(0,146)\end{array}$ & $\begin{array}{l}1,434^{\star \star *} \\
(0,164)\end{array}$ \\
\hline $\begin{array}{l}\text { Revenus habitants < Q1 (= } 12492 € \text { ) } \\
\text { (Ref. : Q1-Q3) }\end{array}$ & $\begin{array}{c}0,925 \\
(0,100)\end{array}$ & $\begin{array}{l}1,102 \\
(0,097)\end{array}$ & $\begin{array}{c}1,066 \\
(0,098)\end{array}$ \\
\hline $\begin{array}{l}\text { Revenus habitants > Q3 (= } 13960 €) \\
\text { (Ref. : Q1-Q3) }\end{array}$ & $\begin{array}{l}1,536^{\star \star \star} \\
(0,191)\end{array}$ & $\begin{array}{l}1,293^{\star \star *} \\
(0,126)\end{array}$ & $\begin{array}{l}1,225^{\star \star} \\
(0,123)\end{array}$ \\
\hline $\begin{array}{l}\text { Taux de chômage > Q3 (= 11,6 \%) } \\
\text { (Ref. : < Q3) }\end{array}$ & $\begin{array}{l}1,118 \\
(0,158)\end{array}$ & $\begin{array}{c}0,872 \\
(0,100)\end{array}$ & $\begin{array}{l}1,115 \\
(0,134)\end{array}$ \\
\hline $\begin{array}{l}\text { Prix du m² des appart < Q1 (= } 1732 € \text { ) } \\
\text { (Ref. : Q1-Q3) }\end{array}$ & $\begin{array}{l}0,881 \\
(0,140)\end{array}$ & $\begin{array}{l}1,084 \\
(0,113)\end{array}$ & $\begin{array}{l}1,154 \\
(0,122)\end{array}$ \\
\hline $\begin{array}{l}\text { Prix du } \mathrm{m}^{2} \text { des appart > Q3 }(=1919 €) \\
\text { (Ref. : Q1-Q3) }\end{array}$ & $\begin{array}{l}1,228^{\star \star} \\
(0,126)\end{array}$ & $\begin{array}{c}1,144 \\
(0,098)\end{array}$ & $\begin{array}{c}0,946 \\
(0,091)\end{array}$ \\
\hline $\begin{array}{l}\text { Densité de Med Génér. < Q1 (= 94) } \\
\text { (Ref. : Q1-Q3) }\end{array}$ & - & $\begin{array}{l}0,853^{*} \\
(0,078)\end{array}$ & $\begin{array}{l}1,044 \\
(0,119)\end{array}$ \\
\hline $\begin{array}{l}\text { Densité de Med Génér. > Q3 (= 108) } \\
\text { (Ref. : Q1-Q3) }\end{array}$ & - & $\begin{array}{l}1,089 \\
(0,193)\end{array}$ & $\begin{array}{l}1,281 \\
(0,236)\end{array}$ \\
\hline $\begin{array}{l}\text { Densité de Med Spéc. < Q1 (= 66) } \\
\text { (Ref. : Q1-Q3) }\end{array}$ & - & $\begin{array}{c}0,928 \\
(0,150)\end{array}$ & $\begin{array}{l}1,199 \\
(0,211)\end{array}$ \\
\hline $\begin{array}{l}\text { Densité de Med Spéc. > Q3 (= 94) } \\
\text { (Ref. : Q1-Q3) }\end{array}$ & - & $\begin{array}{l}1,488^{\star \star \star} \\
(0,191)\end{array}$ & $\begin{array}{l}0,889 \\
(0,162)\end{array}$ \\
\hline $\begin{array}{l}\text { Part + } 75 \text { ans }>\text { Q3 (= 14,2 \%) } \\
\text { (Ref. : < Q3) }\end{array}$ & - & $\begin{array}{c}1,124 \\
(0,134)\end{array}$ & $\begin{array}{l}1,106 \\
(0,134)\end{array}$ \\
\hline $\begin{array}{l}\text { Part hab. comm. rurales > Q3 (= } 30 \%) \\
\text { (Ref. : < Q3) }\end{array}$ & - & $\begin{array}{l}1,242^{\star *} \\
(0,120)\end{array}$ & $\begin{array}{l}1,225^{\star *} \\
(0,121)\end{array}$ \\
\hline $\begin{array}{l}\text { Indicatrice Littoral } \\
\text { (Ref. : aucune commune littorale) }\end{array}$ & - & $\begin{array}{l}0,621^{\star \star *} \\
(0,057)\end{array}$ & $\begin{array}{l}0,680^{\star * *} \\
(0,066)\end{array}$ \\
\hline $\begin{array}{l}\text { Nbre d'heures de soleil < Q1 (= } 1651) \\
\text { (Ref. : Q1-Q3) }\end{array}$ & - & $\begin{array}{l}1,371^{\star *} \\
(0,198)\end{array}$ & $\begin{array}{c}0,795 \\
(0,155)\end{array}$ \\
\hline $\begin{array}{l}\text { Nbre d'heures de soleil > Q3 (= } 1893) \\
\text { (Ref. : Q1-Q3) }\end{array}$ & - & $\begin{array}{l}0,540^{\star \star \star} \\
(0,062)\end{array}$ & $\begin{array}{l}0,695^{\star \star \star} \\
(0,095)\end{array}$ \\
\hline Pseudo $\mathrm{R}^{2}$ & 0,088 & 0,081 & 0,055 \\
\hline Log vraisemblance & -3987 & -4017 & -3305 \\
\hline$\%$ de prédictions correctes & 74 & 75 & 77 \\
\hline Observations & 7567 & 7567 & 6538 \\
\hline
\end{tabular}

Lecture : le tableau présente la valeur des odd-ratios. Le coefficient de 1,348 de la variable « thèse avant 30 ans » (1ère colonne) signifie que, pour les médecins qui ont soutenu leur thèse avant 30 ans, la probabilité de changer de région à l'issue de leur thèse rapportée à celle de ne pas changer de région est 1,348 fois plus élevée que pour les médecins ayant soutenu leur thèse entre 30 et 32 ans (catégorie de référence). ${ }^{* *}$ signifie que l'odd-ratio est significatif au seuil de $1 \%$, ${ }^{*}$ au seuil de $5 \%$ et ${ }^{\star}$ au seuil de $10 \%$; les écarts types figurent entre parenthèses sous les odd-ratios.

Champ : médecins généralistes libéraux de secteur 1, installés pour la première fois en libéral entre 1997 et 2002.

Source : échantillon de médecins généralistes débutants (CNAMTS). 
la densité de médecins spécialistes y est très élevée plutôt que moyennement élevée. Généralistes et spécialistes semblent alors plus substituables que complémentaires: exercer dans une région où la densité de spécialistes est élevée aurait un impact négatif sur la productivité des généralistes (il n'y aurait pas de " partage des tâches ») et sur le nombre de patients potentiels s'adressant à eux. Lorsque l'on prend en compte la variable de revenu, la densité de médecins généralistes n'a en revanche qu'un impact très limité sur la probabilité relative de changer de région à l'issue de la thèse. Mais ces coefficients sont essentiellement dus à l'influence des caractéristiques particulières de l'île-de-France (et notamment à sa forte densité de médecins spécialistes) : lorsque cette région est exclue de l'estimation, les coefficients associés aux variables de densité deviennent non significatifs.

Les effets fixes temporels ne sont jamais significatifs : la probabilité relative, pour un médecin, de quitter sa région de thèse pour s'installer dans une autre région est constante sur la période 1997-2002. Autrement dit, le comportement de sédentarité ne s'est ni accru, ni atténué au cours des années.

Par ailleurs, le sexe n'a aucune influence significative sur la probabilité relative de changer de région: les femmes sont tout aussi mobiles que les hommes à l'issue de leur thèse.

Enfin, on observe que pour les médecins qui ont obtenu leur thèse à un âge relativement jeune (avant 30 ans), la probabilité relative de quitter leur région de thèse est 1,35 fois plus élevée que pour ceux qui ont obtenu leur thèse entre 30 et 32 ans. Ce résultat tient probablement au fait qu'ils ont moins de contraintes familiales que les étudiants plus âgés. Sur la base de cette interprétation, l'âge à la thèse pourrait être utilisé comme un proxy de la situation familiale du médecin, que nous ne connaissons pas. En outre, les médecins qui s'installent tardivement (après 39 ans) ont deux fois plus de chance que ceux qui s'installent à un âge moyen compris entre 32 et 38 ans de changer de région pour s'installer en libéral. Ces médecins sont généralement des médecins qui ont effectué plusieurs années de remplacements avant de s'installer, et probablement dans des régions autres que celles de leur thèse.
Quels facteurs influencent le plus le choix
de la région d'installation d'un médecin ?

Nous nous intéressons, dans un second temps, aux comportements de localisation des médecins qui choisissent de changer de région à l'issue de leur thèse, afin de comprendre les facteurs qui influencent le plus le choix d'une région d'exercice (équation 2$)^{7}$.

\section{Des différences marquées \\ dans le degré d'attractivité des différentes régions françaises}

Les effets fixes régionaux estimés (cf. carte 3 et tableau 3) reflètent l'attractivité des différentes régions françaises pour les médecins qui choisissent de changer de région à l'issue de leur thèse, c'est-à-dire principalement pour les médecins ayant fait leurs études en Île-deFrance ou dans le Limousin, mais aussi dans les régions du centre de la France (régions de formation mises en évidence sur la carte 2). Les régions du Sud de la France apparaissent très attractives. Si l'on met en regard ces résultats avec l'observation de la carte 1, les comportements des nouveaux médecins ne semblent pas différer des choix de localisation réalisés par les médecins des générations plus anciennes. Les fortes disparités de densité entre le Nord et le Sud ne devraient donc pas se résorber naturellement dans les années à venir.

Nos données comportent une limite importante. En effet, certaines régions sont plus exportatrices que d'autres, et l'Île-de-France en particulier (cf. carte 2). Le changement de région observé à l'issue de la thèse pourrait ne pas refléter le choix d'exercer dans une autre région que celle de la thèse mais serait la manifestation d'un retour vers sa région d'origine. Nous ne connaissons pas l'origine géographique des médecins (lieu de naissance ou lieu d'obtention

\footnotetext{
7. Dans notre modélisation, et étant donnée la méthode d'estimation utilisée, nous devons supposer que les médecins choisissent leur région d'installation parmi un ensemble de 22 régions possibles, identique pour tous. Or la région de thèse de chacun de ces médecins ne devrait pas faire partie de leur ensemble de choix; chaque médecin ne devrait être confronté qu'à un choix parmi 21 régions, cet ensemble de 21 régions n'étant pas identique pour tous. Toutefois, notre modélisation repose sur la validité de l'hypothèse d'indépendance des alternatives non pertinentes (cf. annexe) : enlever une région à l'ensemble de choix des médecins ne doit pas modifier les rapports de probabilités de choix de l'ensemble des autres régions. Autrement dit, même si nous supprimions un choix possible à chacun des médecins, les résultats obtenus ne devraient pas être modifiés.
} 
du baccalauréat), une variable qui permettrait de répondre à cette critique. Les données agrégées du Ministère de l'éducation nationale sur l'origine géographique des étudiants en médecine formés dans chaque région fournissent toutefois des éléments d'information sur cette question $^{8}$. Ainsi, $75 \%$ des étudiants en Île-de-France sont originaires de cette région. Parmi les $25 \%$ venant de province, un quart vient des régions voisines (académies d'Amiens, Reims, Rouen ou Tours) ; les trois quarts restant viennent uniformément de l'ensemble des autres régions françaises. Il n'y a donc pas de surreprésentation des étudiants originaires du Sud de la France qui pourrait expliquer le choix massif de ces régions par les étudiants à l'issue de leur thèse. Le même constat est fait pour le Limousin, deuxième région la plus exportatrice d'étudiants en médecine.
Réciproquement, les régions du Sud et de l'Ouest de la France sont très peu exportatrices d'étudiants (cf. carte 2). Il est possible que certains étudiants fassent le choix, dès le début de leurs études de médecine, de se former dans la région dans laquelle ils espèrent, plus tard, exercer. Dans ce cas, l'absence de changement de région à l'issue de la thèse refléterait un choix de changement de région en amont, entre le lycée et la faculté de médecine. Les données agrégées ne valident toutefois pas cette hypothèse : en PACA par exemple, $86 \%$ des

8. Ces données sont disponibles, pour chaque année de 1997 à 2002, dans la base documentaire du service de documentation statistique de la Direction de l'évaluation, de la prospective et de la performance (Depp), Ministère de l'éducation nationale et Ministère de l'enseignement supérieur et de la recherche (http ://www.infocentre.education.fr/acadoc//).

\section{Carte 3}

\section{Estimation du modèle de choix de la région d'installation (équation 2)}

Valeur des effets spécifiques régionaux


Lecture : la valeur des effets fixes estimés figure entre parenthèses sous le nom des régions. Par exemple, les médecins préfèrent, toutes choses égales par ailleurs, s'installer dans les régions PACA ou Languedoc-Roussillon (coefficient positif) plutôt que dans la région de référence (Picardie). Au contraire, les caractéristiques des régions Limousin ou Franche-Comté les rendent moins attractives pour les médecins que la région de référence (coefficient négatif).

Champ : médecins généralistes libéraux de secteur 1, installés pour la première fois en libéral entre 1997 et 2002.

Source : échantillon de médecins généralistes débutants (CNAMTS). 
étudiants en médecine sont originaires de cette région et $10 \%$ sont originaires des régions limitrophes.

\section{Le revenu espéré influence fortement les décisions des médecins qui choisissent de changer de région}

Le revenu des médecins généralistes est introduit sous forme quadratique. Quelle que soit la spécification, les signes des coefficients estimés montrent que l'effet du revenu est croissant à taux décroissant : la probabilité qu'un médecin choisisse une région augmente avec le revenu, mais de moins en moins fortement.

Les moyennes des effets marginaux obtenus pour quelques régions représentatives (cf. tableau 4) facilitent l'interprétation de l'effet du revenu (cf. annexe pour la méthodologie de calcul de ces effets marginaux).

Tableau 3

Estimation du modèle de choix de la région d'installation (équation 2)

Valeur des coefficients estimés

\begin{tabular}{|c|c|c|}
\hline & $\begin{array}{c}\text { Équation 2-a } \\
\text { (avec effets fixes régionaux) }\end{array}$ & $\begin{array}{c}\text { Équation 2-b } \\
\text { (sans effets fixes régionaux) }\end{array}$ \\
\hline Effets fixes régionaux & cf. carte 3 & - \\
\hline Revenu des généralistes (/ 1000) & $\begin{array}{l}0,128^{\star *} \\
(0,052)\end{array}$ & $\begin{array}{l}0,114^{\star \star \star} \\
(0,049)\end{array}$ \\
\hline Revenu des généralistes au carré (/ 1000) & $\begin{array}{c}-0,00099^{\star \star} \\
(0,00046)\end{array}$ & $\begin{array}{c}-0,00079^{\star \star} \\
(0,00044)\end{array}$ \\
\hline Densité de médecins généralistes partant à la retraite & $\begin{array}{c}-0,311^{\star *} \\
(0,125)\end{array}$ & $\begin{array}{c}-0,304^{\star \star \star} \\
(0,119)\end{array}$ \\
\hline Densité des médecins généralistes partant à la retraite au carré & $\begin{array}{l}0,039 \text { * } \\
(0,024)\end{array}$ & $\begin{array}{c}0,027 \\
(0,023)\end{array}$ \\
\hline Taux de chômage & $\begin{array}{r}-0,045 \\
(0,081)\end{array}$ & $\begin{array}{c}-0,049 \\
(0,044)\end{array}$ \\
\hline Prix du $\mathrm{m}^{2}$ des appartements & $\begin{array}{c}0,00025 \\
(0,00033)\end{array}$ & $\begin{array}{c}-0,00013 \\
(0,00028)\end{array}$ \\
\hline Revenu des habitants & $\begin{array}{r}-0,00006 \\
(0,00009)\end{array}$ & $\begin{array}{c}-0,00015^{\star *} \\
(0,00007)\end{array}$ \\
\hline Nombre d'heures de soleil (/ 1000) & - & $\begin{array}{l}0,935^{\star \star \star} \\
(0,415)\end{array}$ \\
\hline Nombre de Rotary Clubs & - & $\begin{array}{l}0,025^{\star \star \star} \\
(0,004)\end{array}$ \\
\hline Densité de médecins généralistes & - & $\begin{array}{c}-0,339^{\star \star \star} \\
(0,117)\end{array}$ \\
\hline Densité de médecins généralistes au carré & - & $\begin{array}{c}0,0014^{\star \star \star} \\
(0,0006)\end{array}$ \\
\hline Densité de médecins spécialistes & - & $\begin{array}{l}0,136^{\star \star \star *} \\
(0,028)\end{array}$ \\
\hline Densité de médecins spécialistes au carré & - & $\begin{array}{l}-0,0007^{\star \star \star} \\
(0,0001)\end{array}$ \\
\hline Taux d'équipement & - & $\begin{array}{c}0,0017 \\
(0,0027)\end{array}$ \\
\hline Part des plus de 75 ans & - & $\begin{array}{r}0,169^{\star} \\
(0,098)\end{array}$ \\
\hline Région avec un littoral & - & $\begin{array}{l}0,605^{\star \star \star} \\
(0,138)\end{array}$ \\
\hline Part population en commune rurale & - & $\begin{array}{l}0,0097 \\
(0,012)\end{array}$ \\
\hline Nombre de chambres d'hôtels / habitant & - & $\begin{array}{c}-0,014 \\
(0,025)\end{array}$ \\
\hline Observations & \multicolumn{2}{|c|}{2178} \\
\hline
\end{tabular}

Lecture : un coefficient positif (exemple : "revenu des généralistes ») signifie que les médecins ont plus de chances de s'installer dans des régions dans lesquelles le revenu des médecins est élevé ; les écarts types figurent entre parenthèses sous les coefficients estimés ; *** signifie que le coefficient est significatif au seuil de $1 \%,{ }^{* *}$ au seuil de $5 \%$ et * au seuil de $10 \%$; les effets des variables spécifiques au médecin (sexe et âge à l'installation, soit 66 coefficients) ne sont pas reportés dans ce tableau par souci de lisibilité. Notre modèle n'inclut pas d'indicatrices temporelles : nous supposons que l'attractivité des différentes régions est constante sur la période 1997-2002. En effet, cela impliquerait d'estimer 105 coefficients supplémentaires (5 dummies temporelles pour chacune des 21 régions), ce qui rend le modèle difficile à estimer. Toutefois, la plage temporelle de nos données est suffisamment faible pour admettre cette hypothèse. Champ : médecins généralistes libéraux de secteur 1, installés pour la première fois en libéral entre 1997 et 2002.

Source : échantillon de médecins généralistes débutants (CNAMTS). 
Toutes choses égales par ailleurs, les médecins changent de région pour s'installer dans des régions où le revenu est élevé. En effet, pour ces médecins qui font le choix de déménager à l'issue de leur thèse, la qualité de vie peut diminuer (en raison des différents coûts psychologiques et financiers associés à la mobilité géographique): s'installer dans des régions qui procurent des revenus plus élevés leur permet de compenser ces coûts' ${ }^{9}$. Les effets marginaux indiquent que la probabilité moyenne de s'installer en région PACA, le revenu des autres régions restant constant, augmente de 0,035 à 0,038 points lorsque le revenu de cette région augmente de 10000 euros annuels (soit une hausse moyenne de revenus de $22 \%$ ). En termes de pourcentages, la probabilité de s'installer en PACA passerait donc de $11 \%$ à $14,5 \%$ ou $14,8 \%$. L'effet du revenu est plus faible dans les autres régions, il est même négatif (mais dans ce cas, l'effet marginal est non significatif) dans les régions dans lesquelles les revenus moyens sont les plus élevés. Ainsi, cette même hausse de 10000 annuels dans la région du Nord de la France conduirait à une variation de la probabilité de s'y installer comprise entre $-0,0003$ et $+0,001$ points de pourcentage. Toutes choses égales par ailleurs (et notamment à conditions de vie identiques), les médecins sont donc d'autant plus sensibles à une hausse du revenu que leurs revenus sont faibles. Ces résultats ne sont pas contradictoires avec notre intuition initiale selon laquelle les médecins effectueraient, au moment de leur installation, un arbitrage entre revenu et qualité de vie, approximée ici par le nombre d'heures de soleil (cf. graphique) : il s'agit ici d'un effet du revenu mesuré toutes choses égales par ailleurs, et notamment à niveau d'ensoleillement identique.

Cette spécification ne permet pas de savoir si les médecins valorisent différemment le revenu en fonction de la région dans laquelle ils s'installent. Pour cela, il faudrait que l'effet du revenu dépende de la région d'installation. Cette régression, qui nécessiterait l'estimation de 42 coefficients, rendrait complexe l'interprétation de ces effets différenciés. Nous avons donc opté pour une voie médiane dans laquelle nous autorisons une hétérogénéité de l'effet du revenu en fonction de trois catégories de régions : les régions dans lesquelles la densité de médecins généralistes est inférieure ou égale au $1^{\text {er }}$ quintile, celles où elle est comprise entre le $1^{\text {er }}$ et le $5^{\text {ème }}$ quintile et celles où elle est supérieure ou égale au $5^{\text {ème }}$ quintile

9. La même régression, effectuée sur les médecins qui ne changent pas de région à l'issue de leur thèse (les résultats ne sont pas présentés ici), conduit à un effet non significatif du revenu : le revenu n'est un facteur déterminant du choix de localisation des médecins que pour ceux qui changent de région à l'issue de leur thèse.

\begin{tabular}{|c|c|c|c|c|c|c|c|}
\hline \multirow[b]{2}{*}{ Région } & \multirow[b]{2}{*}{$\begin{array}{c}\text { Densité } \\
\text { moyenne }\end{array}$} & \multirow[b]{2}{*}{$\begin{array}{l}\text { Revenu } \\
\text { moyen }\end{array}$} & \multirow[b]{2}{*}{$\begin{array}{c}p_{j} \\
(\%)\end{array}$} & \multicolumn{2}{|c|}{$\begin{array}{l}\text { Effets marginaux du revenu } \\
\text { (équation 2) }\end{array}$} & \multicolumn{2}{|c|}{$\begin{array}{c}\text { Effets marginaux du revenu } \\
\text { (équation 2' avec effet différencié } \\
\text { du revenu) }\end{array}$} \\
\hline & & & & $\begin{array}{l}\text { Régression avec } \\
\text { effets fixes }\end{array}$ & $\begin{array}{l}\text { Régression sans } \\
\text { effets fixes }\end{array}$ & $\begin{array}{l}\text { Régression avec } \\
\text { effets fixes }\end{array}$ & $\begin{array}{l}\text { Régression sans } \\
\text { effets fixes }\end{array}$ \\
\hline PACA & 130 & 45878 & 11 & $\begin{array}{c}0,035 \\
{[0,021 ; 0,048]}\end{array}$ & $\begin{array}{c}0,038 \\
{[0,027 ; 0,051]}\end{array}$ & $\begin{array}{c}0,039 \\
{[0,024 ; 0,054]}\end{array}$ & $\begin{array}{c}0,028 \\
{[0,001 ; 0,056]}\end{array}$ \\
\hline Bretagne & 101 & 51127 & 6,6 & $\begin{array}{c}0,016 \\
{[0,008 ; 0,023]}\end{array}$ & $\begin{array}{c}0,018 \\
{[0,009 ; 0,007]}\end{array}$ & $\begin{array}{c}0,013 \\
{[0,004 ; 0,022]}\end{array}$ & $\begin{array}{c}0,018 \\
{[0,006 ; 0,029]}\end{array}$ \\
\hline Île-de-France & 94 & 50849 & 4,4 & $\begin{array}{c}0,011 \\
{[0,004 ; 0,017]}\end{array}$ & $\begin{array}{c}0,014 \\
{[0,007 ; 0,02]}\end{array}$ & $\begin{array}{c}0,009 \\
{[0,006 ; 0,013]}\end{array}$ & $\begin{array}{c}0,017 \\
{[0,006 ; 0,029]}\end{array}$ \\
\hline $\begin{array}{l}\text { Champagne- } \\
\text { Ardenne }\end{array}$ & 91 & 66107 & 2,6 & $\begin{array}{c}-0,0003 \\
{[-0,006 ; 0,005]}\end{array}$ & $\begin{array}{c}0,002 \\
{[-0,003 ; 0,007]}\end{array}$ & $\begin{array}{c}0,002 \\
{[-0,003 ; 0,008]}\end{array}$ & $\begin{array}{c}0,005 \\
{[-0,002 ; 0,012]}\end{array}$ \\
\hline Nord & 103 & 66371 & 1,9 & $\begin{array}{c}-0,0003 \\
{[-0,004 ; 0,003]}\end{array}$ & $\begin{array}{c}0,001 \\
{[-0,002 ; 0,005]}\end{array}$ & $\begin{array}{c}-0,003 \\
{[-0,007 ; 0,007]}\end{array}$ & $\begin{array}{c}-0,002 \\
{[-0,007 ; 0,002]}\end{array}$ \\
\hline
\end{tabular}

Lecture : en plus de la valeur des effets marginaux, le tableau fournit la densité moyenne de médecins généralistes dans les régions sélectionnées, le revenu moyen et la probabilité non conditionnelle $\left(p_{j}\right)$ de choisir chacune de ces régions. Pour calculer les effets marginaux associés à l'équation 2, on utilise les coefficients du tableau 3 ; les résultats des estimations de l'équation 2' nécessaires au calcul des effets marginaux n'étant pas présentés, les coefficients estimés des variables de revenus obtenus dans le cadre de l'estimation sans effets fixes régionaux sont les suivants : coefficient du revenu et du revenu au carré $=0,165$ et - 0,0011 pour les régions à faible densité, 0,190 et - 0,0015 pour les régions de densité moyenne et 0,212 et -0,0019 pour les régions de forte densité. L'intervalle de confiance à $95 \%$ figure sous les effets marginaux.

Champ : médecins généralistes libéraux de secteur 1, installés pour la première fois en libéral entre 1997 et 2002.

Source : échantillon de médecins généralistes débutants (CNAMTS). 
(cette nouvelle équation est appelée équation 2'). Comme précédemment, l'effet du revenu est introduit sous forme quadratique. Pour ne pas alourdir la présentation, seuls les effets marginaux du revenu obtenus à partir de cette régression sont reportés (cf. tableau 4) : l'effet du revenu reste supérieur dans les régions dans lesquelles la densité est élevée (et donc dans lesquelles le niveau moyen de revenu est plus faible). Toutes choses égales par ailleurs, les médecins exerçant dans les régions où le niveau moyen de revenu est faible valorisent plus fortement le revenu que les médecins exerçant dans les autres régions. La prise en compte d'un effet différencié du revenu montre toutefois que les résultats précédents tendent à surestimer l'impact du revenu pour les médecins qui exercent dans les régions où la densité est élevée et à le sous-estimer légèrement (même si le coefficient reste non significatif) pour ceux qui exercent dans les régions où la densité de médecins est faible.

Mais entre niveau de revenu et qualité de vie, quel facteur importe le plus pour les médecins? Nous aborderons cette question dans la suite de l'article.

\section{Les politiques financières peuvent-elles alors influencer les comportements de localisation des médecins?}

Les pouvoirs publics ont récemment mis en place des politiques financières incitatives à l'installation dans des zones sous-dotées en médecins. Les estimations réalisées précédemment peuvent être exploitées afin de simuler l'impact de ces politiques financières sur les comportements de localisation des médecins : si, comme nous venons de le voir, le revenu espéré influence en partie les choix d'installation des médecins, dans quelle mesure les aides financières permettraient-elles de modifier la répartition des médecins?

En utilisant les estimations qui autorisent un effet différencié du revenu selon les régions, nous simulons l'impact, sur la répartition des médecins, de l'attribution d'une « prime à l'installation » (de 5000 ou de 10000 euros annuels) à tous les médecins qui choisissent de s'installer dans une région à faible densité médicale ${ }^{10}$ (cf. tableau 5). Nous comparons ensuite la répartition observée des médecins entre les régions, à la répartition simulée en fonction de ces deux politiques (cf. annexe pour la description de la méthodologie).
Cette simulation est effectuée sur les seuls médecins qui changent de région après leur thèse. De ce fait, elle ne permet pas d'analyser l'impact de la prime à l'installation sur les comportements des médecins qui étaient initialement non mobiles. Les résultats des estimations de l'équation (1) nous imposent de raisonner ainsi. En effet, examiner les comportements en population générale nécessiterait de modéliser, dans un premier temps, l'impact du revenu (de nouveau différencié en trois tranches en fonction de la densité) sur la probabilité de changer de région à l'issue de la thèse. Or, dans cette régression, les différentes variables de revenus ne sont pas significatives. Il est donc ensuite impossible de mesurer comment cette probabilité est modifiée lorsque le revenu de certaines régions augmente, et donc de simuler la politique de prime à l'installation sur l'ensemble de la population des médecins. Ce résultat signifie toutefois que les comportements des médecins non mobiles ne devraient pas être influencés par la politique de prime à l'installation : ces derniers continueront à s'installer dans la région de leur thèse.

La prime à l'installation de 5000 euros (l'équivalent d'un revenu mensuel), attribuée aux médecins s'installant dans les cinq régions les moins densément peuplées, conduirait à une augmentation de $9 \%$ du nombre de médecins exerçant dans le Centre, de $8 \%$ en BasseNormandie, de $2 \%$ en Île-de-France et de $1,7 \%$ en Champagne-Ardenne (cf. tableau 5). Seul le nombre de médecins s'installant en Picardie diminuerait $(-1,6 \%)$. La Picardie est la région française dans laquelle les médecins ont les revenus annuels les plus élevés (cf. graphique) : les niveaux de revenus sont donc suffisamment élevés pour que la prime ne soit pas un élément déterminant à l'installation dans ces zones. Pour les quatre autres régions, l'effet de la prime serait non négligeable : grâce au supplément de revenu, ces régions deviendraient suffisamment attractives pour que de nouveaux médecins décident de s'y installer, même si, quantitativement, l'effet peut paraître limité au regard du nombre de médecins déjà présents dans la région. Avec une prime de 5000 euros, environ 19 médecins supplémentaires s'installeraient dans ces régions. Mais ce mécanisme incitatif profiterait également

10. Plus qu'une prime à l'installation, il s'agit ici d'un supplément annuel de revenu attribué sur l'ensemble de la carrière du médecin s'il choisit de s'installer dans une des régions concernées par ce mécanisme incitatif. 
aux médecins spontanément installés dans ces régions et pour lesquels il s'agit d'un pur effet d'aubaine. Pour l'assurance maladie, le coût total de cette mesure s'élèverait donc à environ 2,2 millions d'euros soit $0,04 \%$ du montant des consultations d'omnipraticiens

Tableau 5

Modification de la répartition des médecins sur le territoire suite à la mise en place d'une politique de prime à l'installation (régions classées par ordre croissant de la densité en 2002)

\begin{tabular}{|c|c|c|c|c|c|c|c|}
\hline Région & $\begin{array}{l}\text { Densité } \\
\text { en } 2002\end{array}$ & $\begin{array}{c}\text { Nombre } \\
\text { de méde- } \\
\text { cins installés } \\
\text { (= nombre } \\
\text { simulé sans } \\
\text { prime) }\end{array}$ & $\begin{array}{c}\text { Instauration } \\
\text { d'une prime à } \\
\text { l'installation? }\end{array}$ & $\begin{array}{c}\text { Nombre } \\
\text { de médecins } \\
\text { simulé avec } \\
\text { une prime } \\
\text { de } 5000 € \\
\text { (variation en \%) }\end{array}$ & $\begin{array}{c}\text { Densité suite } \\
\text { à la prime de } \\
5000 € \\
\text { (variation en \%) }\end{array}$ & \begin{tabular}{|c} 
Nombre \\
de médecins \\
simulé avec \\
une prime \\
de $10000 €$ \\
(variation en \%)
\end{tabular} & $\begin{array}{c}\text { Densité suite } \\
\text { à la prime de } \\
10000 € \\
\text { (variation } \\
\text { en } \% \text { ) }\end{array}$ \\
\hline Centre & 88,76 & 131 & OUI & $\begin{array}{c}142,5 \\
(+8,78)\end{array}$ & $\begin{array}{c}89,22 \\
(+0,52)\end{array}$ & $\begin{array}{c}149,5 \\
(+14,12)\end{array}$ & $\begin{array}{c}89,51 \\
(+0,84)\end{array}$ \\
\hline Picardie & 89,79 & 75 & OUI & $\begin{array}{c}73,8 \\
(-1,64)\end{array}$ & $\begin{array}{c}89,73 \\
(-0,07)\end{array}$ & $\begin{array}{c}70 \\
(-6,70)\end{array}$ & $\begin{array}{c}89,53 \\
(-0,29)\end{array}$ \\
\hline $\begin{array}{l}\text { Basse } \\
\text { Normandie }\end{array}$ & 90,48 & 63 & OUI & $\begin{array}{c}68 \\
(+7,89)\end{array}$ & $\begin{array}{c}90,83 \\
(+0,39)\end{array}$ & $\begin{array}{c}70,7 \\
(+12,20)\end{array}$ & $\begin{array}{c}91,02 \\
(+0,59)\end{array}$ \\
\hline Île-de-France & 91,69 & 97 & OUI & $\begin{array}{c}99 \\
(+2,06)\end{array}$ & $\begin{array}{c}91,72 \\
(+0,03)\end{array}$ & $\begin{array}{c}100,9 \\
(+4,02)\end{array}$ & $\begin{array}{c}91,73 \\
(+0,05)\end{array}$ \\
\hline $\begin{array}{l}\text { Champagne- } \\
\text { Ardenne }\end{array}$ & 91,38 & 56 & OUI & $\begin{array}{c}56,9 \\
(+1,69)\end{array}$ & $\begin{array}{c}91,45 \\
(+0,08)\end{array}$ & $\begin{array}{c}55,9 \\
(-0,19)\end{array}$ & $\begin{array}{c}91,38 \\
(-0,004)\end{array}$ \\
\hline $\begin{array}{l}\text { Haute- } \\
\text { Normandie }\end{array}$ & 93,25 & 57 & NON & $\begin{array}{c}56,7 \\
(-0,53)\end{array}$ & $\begin{array}{c}93,23 \\
(-0,02)\end{array}$ & $\begin{array}{c}56,1 \\
(-1,59)\end{array}$ & $\begin{array}{c}93,20 \\
(-0,05)\end{array}$ \\
\hline Pays de la Loire & 93,95 & 144 & NON & $\begin{array}{c}142,5 \\
(-1,04)\end{array}$ & $\begin{array}{c}93,90 \\
(-0,05)\end{array}$ & $\begin{array}{c}142 \\
(-1,39)\end{array}$ & $\begin{array}{c}93,88 \\
(-0,07)\end{array}$ \\
\hline Lorraine & 95,82 & 44 & NON & $\begin{array}{c}43,5 \\
(-1,10)\end{array}$ & $\begin{array}{c}95,80 \\
(-0,02)\end{array}$ & $\begin{array}{c}43,3 \\
(-1,59)\end{array}$ & $\begin{array}{c}95,79 \\
(-0,03)\end{array}$ \\
\hline Bourgogne & 96,14 & 64 & NON & $\begin{array}{c}63,3 \\
(-1,09)\end{array}$ & $\begin{array}{c}96,10 \\
(-0,04)\end{array}$ & $\begin{array}{c}63,1 \\
(-1,41) \\
\end{array}$ & $\begin{array}{c}96,09 \\
(-0,05)\end{array}$ \\
\hline Rhône-Alpes & 99,03 & 219 & NON & $\begin{array}{c}216,7 \\
(-1,05)\end{array}$ & $\begin{array}{c}98,99 \\
(-0,04)\end{array}$ & $\begin{array}{c}215,9 \\
(-1,42)\end{array}$ & $\begin{array}{c}98,98 \\
(-0,05)\end{array}$ \\
\hline Bretagne & 100,74 & 144 & NON & $\begin{array}{c}142,5 \\
(-1,04)\end{array}$ & $\begin{array}{l}100,69 \\
(-0,04)\end{array}$ & $\begin{array}{c}141,9 \\
(-1,45)\end{array}$ & $\begin{array}{l}100,67 \\
(-0,06)\end{array}$ \\
\hline Franche-Comté & 101,63 & 25 & NON & $\begin{array}{c}24,7 \\
(-1,20)\end{array}$ & $\begin{array}{l}101,61 \\
(-0,02)\end{array}$ & $\begin{array}{c}24,7 \\
(-1,2)\end{array}$ & $\begin{array}{l}101,61 \\
(-0,02)\end{array}$ \\
\hline Auvergne & 102,89 & 59 & NON & $\begin{array}{c}58,4 \\
(-1,02) \\
\end{array}$ & $\begin{array}{l}102,85 \\
(-0,04) \\
\end{array}$ & $\begin{array}{c}58,2 \\
(-1,36) \\
\end{array}$ & $\begin{array}{l}102,83 \\
(-0,05) \\
\end{array}$ \\
\hline $\begin{array}{l}\text { Nord-Pas-de- } \\
\text { Calais }\end{array}$ & 103,65 & 42 & NON & $\begin{array}{c}41,5 \\
(-1,19)\end{array}$ & $\begin{array}{c}103,64 \\
(-0,007)\end{array}$ & $\begin{array}{c}41,3 \\
(-1,6)\end{array}$ & $\begin{array}{l}103,64 \\
(-0,01)\end{array}$ \\
\hline Alsace & 104,18 & 32 & NON & $\begin{array}{c}31,7 \\
(-0,93)\end{array}$ & $\begin{array}{l}104,16 \\
(-0,02)\end{array}$ & $\begin{array}{c}31,6 \\
(-1,25)\end{array}$ & $\begin{array}{l}104,16 \\
(-0,02)\end{array}$ \\
\hline $\begin{array}{l}\text { Poitou- } \\
\text { Charentes }\end{array}$ & 106,54 & 97 & NON & $\begin{array}{c}96 \\
(-1,03)\end{array}$ & $\begin{array}{l}106,48 \\
(-0,06)\end{array}$ & $\begin{array}{c}95,6 \\
(-1,45)\end{array}$ & $\begin{array}{l}106,46 \\
(-0,08)\end{array}$ \\
\hline Corse & 111,27 & 54 & NON & $\begin{array}{c}53,4 \\
(-1,11)\end{array}$ & $\begin{array}{l}111,05 \\
(-0,19)\end{array}$ & $\begin{array}{c}53,2 \\
(-1,48)\end{array}$ & $\begin{array}{l}110,98 \\
(-0,26)\end{array}$ \\
\hline Midi-Pyrénées & 115,67 & 140 & NON & $\begin{array}{c}138,5 \\
(-1,07)\end{array}$ & $\begin{array}{l}115,61 \\
(-0,05)\end{array}$ & $\begin{array}{c}138 \\
(-1,42)\end{array}$ & $\begin{array}{l}115,59 \\
(-0,06)\end{array}$ \\
\hline Aquitaine & 117,67 & 158 & NON & $\begin{array}{c}156,3 \\
(-1,07)\end{array}$ & $\begin{array}{l}117,61 \\
(-0,05)\end{array}$ & $\begin{array}{c}155,7 \\
(-1,45) \\
\end{array}$ & $\begin{array}{l}117,59 \\
(-0,06)\end{array}$ \\
\hline Limousin & 120,08 & 22 & NON & $\begin{array}{c}21,8 \\
(-0,91)\end{array}$ & $\begin{array}{l}120,05 \\
(-0,02)\end{array}$ & $\begin{array}{l}21,7 \\
(1,36)\end{array}$ & $\begin{array}{l}120,04 \\
(-0,04)\end{array}$ \\
\hline $\begin{array}{l}\text { Provence-Alpes- } \\
\text { Côte d'Azur }\end{array}$ & 127 & 240 & NON & $\begin{array}{c}237,6 \\
(-1)\end{array}$ & $\begin{array}{l}126,94 \\
(-0,05)\end{array}$ & $\begin{array}{c}236,8 \\
(-1,34)\end{array}$ & $\begin{array}{l}126,92 \\
(-0,06)\end{array}$ \\
\hline $\begin{array}{l}\text { Languedoc- } \\
\text { Roussillon }\end{array}$ & 129,36 & 215 & NON & $\begin{array}{l}212,8 \\
(-1,02)\end{array}$ & $\begin{array}{l}129,27 \\
(-0,07)\end{array}$ & $\begin{array}{c}212 \\
(-1,39)\end{array}$ & $\begin{array}{l}129,23 \\
(-0,09)\end{array}$ \\
\hline
\end{tabular}

Lecture : une prime à l'installation de $5000 €$ ou de $10000 €$ est instaurée dans les 5 régions les moins densément peuplées en médecins (valeur de la densité de 2002). Dans la région Centre par exemple, une prime de $5000 €$ conduit à ce que 11,5 jeunes médecins supplémentaires s'installent sur la période 1997-2002 (soit une hausse de 8,78\%). Cela conduit à une augmentation de la densité de médecins généralistes dans cette région de 0,52\%.

Champ : médecins généralistes libéraux de secteur 1, installés pour la première fois en libéral entre 1997 et 2002.

Source : échantillon de médecins généralistes débutants (CNAMTS). 
remboursées en $2002^{11}$ ). Si le montant de la prime était doublé, près de 23 médecins supplémentaires s'installeraient dans ces régions, pour un coût total pour l'assurance maladie de près de 4,45 millions d'euros (soit 0,09\% du montant des consultations d'omnipraticiens remboursées en 2002). Au total, ces mesures permettraient d'augmenter la densité médicale de $0,5 \%$ ou $0,8 \%$ dans le Centre et de $0,4 \%$ ou $0,6 \%$ en Basse-Normandie. Si les coûts paraissent faibles au regard de la totalité des dépenses remboursées par l'assurance maladie, ils représentent néanmoins, en raison de l'effet d'aubaine, 100000 à 200000 euros par médecin supplémentaire installé, selon le montant de la prime.

En contrepartie, cette nouvelle répartition diminuerait mécaniquement le nombre de médecins s'installant dans les régions de densité moyenne à élevée. Mais la diminution ne serait pas uniforme et dépendrait fortement de l'attractivité des régions. Ainsi, le nombre de médecins diminuerait de $1,6 \%$ en Picardie mais seulement de $1,2 \%$ en PACA et Languedoc-Roussillon. L'agrément apporté par les conditions de vie dans les différentes régions étant constant, les régions les plus attractives avant cette politique d'incitation financière le demeureraient. En conséquence, une telle politique d'incitation financière n'aboutirait pas à tous les effets désirés : l'incitation financière permettrait de résorber une partie du déséquilibre dans la mesure où les régions les plus déficitaires verraient le nombre de leurs médecins augmenter. Mais le rééquilibrage se ferait au détriment des régions juste à l'équilibre qui perdraient le plus de médecins. En revanche, les régions très excédentaires resteraient en excédent. Les résultats sont encore plus marqués, mais resteraient globalement similaires quand la prime à l'installation est plus élevée, égale à 10000 euros.

Cette simulation présente évidemment des limites. Elle est essentiellement statique et ne prend notamment pas en compte le fait que si les incitations parviennent à attirer des médecins supplémentaires dans des zones moins dotées, la variation de la densité qui en résulterait influencerait les revenus espérés dans cette zone ainsi que les comportements de localisation des autres médecins.

11. Pour la France entière, ce montant s'élève à 4792 millions d'euros (Eco-Santé, 2012).

\section{Au-delà du revenu, les médecins privilégient fortement leur qualité de vie}

Dans la régression, plusieurs variables sont introduites afin de capturer l'influence du contexte économique et sociodémographique, ainsi que des conditions de vie sur le choix d'installation dans les différentes régions.

Le revenu des habitants n'influence pas significativement le choix d'une région d'exercice. Ce résultat n'est pas surprenant dans la mesure où les médecins généralistes de secteur 1 pratiquent des tarifs fixés et, en théorie, peu de dépassements d'honoraires. Le taux de remboursement des patients pour les consultations effectuées chez les généralistes est donc élevé. À la différence des médecins spécialistes, la probabilité de recours à un médecin généraliste ne dépend donc pas du revenu des individus (voir par exemple, Raynaud, 2005). Pour les mêmes raisons, le choix d'une région d'installation ne dépend pas non plus du taux de chômage de la région.

Les comportements des généralistes ne sont pas influencés par les revenus de leurs patients potentiels, mais, en raison du paiement à l'acte qui relie étroitement leurs revenus au niveau de leur activité, ils sont influencés par le nombre potentiel de patients. Deux variables sont, à ce titre, éclairantes. D'une part, la probabilité de choix d'une région dépend négativement du nombre de généralistes déjà installés dans cette région. Toutes choses égales par ailleurs, cela signifie que les médecins sont plus à même de s'installer dans des régions où le nombre de généralistes en activité est faible et donc où la clientèle potentielle y est plus nombreuse. À niveau de revenus et qualité de vie identique, les médecins préfèreront s'installer dans des régions dans lesquelles leur revenu peut être atteint plus facilement. Par ailleurs, les généralistes sont plus à même de s'installer dans des régions dans lesquelles la part des patients de plus de 75 ans est élevée. En effet, le taux de recours aux soins et les dépenses de santé étant croissants avec l'âge, une part élevée de patients âgés se traduit par une demande plus forte qui s'adresse aux médecins. En revanche, la probabilité de choix d'une région dépend positivement du nombre de spécialistes. Ce résultat montre qu'il ne semble pas y avoir de concurrence mais plutôt une complémentarité entre les généralistes et les spécialistes. En outre, ces deux catégories de médecins semblent attirées par l'installation dans les mêmes régions. 
Enfin, la qualité des conditions de vie influence fortement les comportements de localisation des médecins. Trois variables sont significatives dans la régression. Toutes choses égales par ailleurs, la probabilité de s'installer dans une région est une fonction croissante du nombre d'heures de soleil, du nombre de Rotary Clubs dans la région mais également de la présence du littoral. Nous nous intéressons plus particulièrement à la variable d'ensoleillement. Afin de quantifier l'importance de cette variable par rapport à une variable d'ordre économique, comme le revenu espéré, nous calculons le taux marginal de substitution (TMS) entre revenu et ensoleillement dans chaque région. Plus précisément, nous obtenons, pour chaque région, le montant de revenu additionnel nécessaire pour compenser la perte d'une heure de soleil et maintenir une utilité constante (cf. annexe pour une description de la méthode de calcul de ce TMS). Le montant de revenu nécessaire pour compenser la perte d'une heure de soleil est croissant avec le niveau de revenu de la région (autrement dit, il est décroissant avec le nombre d'heures de soleil). Ainsi, le TMS s'élève à 263 pour les médecins exerçant en Picardie (il est nécessaire de leur donner 2630 euros de revenu supplémentaire pour compenser la perte de 10 heures de soleil) mais de seulement 22,5 en PACA. Pour les médecins, la perte d'une unité de soleil est donc d'autant plus valorisée qu'ils exercent dans des régions dans lesquelles le nombre d'heures de soleil est faible.

Toutefois, un calcul plus intéressant consiste à utiliser la valeur de ces TMS régionaux pour calculer le montant de revenu additionnel qu'il serait nécessaire de donner aux médecins qui s'installent dans les régions à forte densité (PACA, Languedoc-Roussillon) ou moyenne densité (Auvergne, Franche-Comté) pour qu'ils modifient leurs choix et acceptent de s'installer dans les régions à faible densité (Centre, Île-de-France et Basse-Normandie); en d'autres termes, il s'agit de calculer le montant de revenu qui leur permettrait de maintenir le même niveau d'utilité malgré la diminution de leur qualité de vie (mesurée ici par la baisse du nombre d'heures de soleil). Ce montant est appelé revenu équivalent (cf. tableau 6).

Tableau 6

Taux marginal de substitution (TMS) entre revenu et ensoleillement pour différentes régions

\begin{tabular}{|c|c|c|c|c|c|c|c|}
\hline & $\begin{array}{l}\text { Différence } \\
\text { de revenu } \\
\text { (euros) }\end{array}$ & $\begin{array}{l}\text { Différence } \\
\qquad \%)\end{array}$ & $\begin{array}{c}\text { Différence } \\
\text { d'ensoleillement } \\
\text { (heures) }\end{array}$ & $\begin{array}{l}\text { Différence } \\
\qquad \%)\end{array}$ & $\begin{array}{l}\text { TMS de la } \\
\text { région } \\
\text { d'origine }\end{array}$ & $\begin{array}{l}\text { Revenu } \\
\text { équivalent } \\
\text { (euros) }\end{array}$ & $\begin{array}{c}\text { Prime } \\
\text { annuelle } \\
\text { à verser } \\
\text { (euros) }\end{array}$ \\
\hline $\begin{array}{l}\text { Provence-Alpes-Côte d'Azur } \rightarrow \\
\text { Centre }\end{array}$ & 12317 & 26,8 & -1163 & $-40,4$ & 22,52 & 26191 & 13874 \\
\hline $\begin{array}{l}\text { Provence-Alpes-Côte d'Azur } \rightarrow \\
\text { Île-de-France }\end{array}$ & 4971 & 10,8 & -1300 & $-45,1$ & 22,52 & 29276 & 24305 \\
\hline $\begin{array}{l}\text { Provence-Alpes-Côte d'Azur } \rightarrow \\
\text { Basse-Normandie }\end{array}$ & 13230 & 28,8 & -1230 & $-42,7$ & 22,52 & 27699 & 4469 \\
\hline Languedoc-Roussillon $\rightarrow$ Centre & 5981 & 11,5 & -792 & $-31,6$ & 29,68 & 23506 & 17525 \\
\hline $\begin{array}{l}\text { Languedoc-Roussillon } \rightarrow \\
\text { Île-de-France }\end{array}$ & -1365 & $-2,6$ & -929 & $-37,0$ & 29,68 & 27572 & 28937 \\
\hline $\begin{array}{l}\text { Languedoc-Roussillon } \rightarrow \\
\text { Basse-Normandie }\end{array}$ & 689 & 13,2 & -859 & $-34,2$ & 29,68 & 25495 & 24806 \\
\hline Auvergne $\rightarrow$ Centre & 5070 & 9,5 & -131 & $-7,1$ & 31,11 & 4075 & -995 \\
\hline Auvergne $\rightarrow$ Île-de-France & -2276 & $-4,3$ & -268 & $-14,5$ & 31,11 & 8337 & 10613 \\
\hline Auvergne $\rightarrow$ Basse-Normandie & 5983 & 11,3 & -198 & $-10,7$ & 31,11 & 6159 & 176 \\
\hline Franche-Comté $\rightarrow$ Centre & 5654 & 10,8 & -47 & $-2,7$ & 30,17 & 1417 & -4237 \\
\hline Franche-Comté $\rightarrow$ Île-de-France & -1692 & $-3,2$ & -184 & $-10,4$ & 30,17 & 5551 & 7209 \\
\hline $\begin{array}{l}\text { Franche-Comté } \rightarrow \\
\text { Basse-Normandie }\end{array}$ & 6567 & 12,5 & -114 & $-6,5$ & 30,17 & 3439 & -3128 \\
\hline
\end{tabular}

Lecture : la 1ère ligne du tableau se lit de la façon suivante: si les médecins choisissaient de s'installer dans le Centre plutôt qu'en PACA, ils gagneraient $12317 €$ annuels supplémentaires (soit une augmentation de $26,8 \%$ de revenu) mais perdraient en revanche 1163 heures d'ensoleillement (soit une diminution de $40 \%$ ). Pour compenser cette perte de qualité de vie, et maintenir la même utilité, étant donné le TMS calculé pour les médecins exerçant en PACA (22,52), ces médecins devraient gagner $26191 €$. La prime à verser à ces médecins pour qu'ils acceptent l'installation dans la région Centre serait donc de $13874 €$.

Champ : médecins généralistes libéraux de secteur 1, installés pour la première fois en libéral entre 1997 et 2002.

Source : échantillon de médecins généralistes débutants (CNAMTS). 
Les médecins qui s'installeraient dans le Centre plutôt qu'en PACA gagneraient 12317 euros annuels supplémentaires (soit une augmentation de $26 \%$ de revenu) mais perdraient en revanche 1163 heures de soleil, soit une diminution de $40 \%$ (cf. tableau 6). Pour compenser cette perte de qualité de vie, et maintenir la même utilité, ces médecins devraient gagner 26191 euros. Ce revenu équivalent est supérieur au supplément de revenu apporté par un exercice dans la région Centre plutôt que la région PACA, expliquant ainsi pourquoi les médecins qui valorisent leur qualité de vie ne s'installent pas spontanément dans cette région du Centre. La différence entre le revenu équivalent et le différentiel de revenu représente la prime qu'il conviendrait de verser aux médecins pour qu'ils acceptent de se localiser dans ces régions moins attractives. Pour que ces médecins acceptent l'installation dans la région Centre, l'état et/ou les collectivités locales devraient mettre en place une prime s'élevant en moyenne à 13874 euros. Pour inciter les médecins à déménager de la région PACA à la région Île-de-France (et qu'ils conservent le même niveau d'utilité), la prime moyenne à leur verser serait en revanche bien supérieure, s'élevant à 24305 euros.

Les primes à verser sont d'un montant inférieur pour les médecins initialement installés dans les régions moins densément peuplées telles que l'Auvergne ou la Franche-Comté. Elles sont même parfois négatives : le supplément de revenu que les médecins auraient en déménageant vers les régions du Centre ou de la BasseNormandie compense suffisamment leur perte de qualité de vie, qui est, il est vrai, beaucoup plus faible que pour les médecins originaires de PACA ou du Languedoc-Roussillon.

$\mathrm{Au}$ total, ces résultats corroborent la simulation de la mise en place d'une politique de prime à l'installation (cf. tableau 5) et permettent de comprendre pourquoi les primes à l'installation n'impactent pas identiquement toutes les régions faiblement densément peuplées : le montant de ces primes ne compense pas toujours suffisamment la perte de qualité de vie consentie par les médecins. Les primes de 5000 euros ou 10000 euros sont suffisamment attractives pour inciter les médecins à s'installer dans des régions telles que la BasseNormandie ou le Centre, notamment lorsqu'ils sont originaires des régions moyennement densément peuplées : le différentiel de revenu, joint à l'existence de primes, compense largement la perte de qualité de vie. Elles ne le sont cependant pas suffisamment pour attirer les médecins venant des régions fortement ensoleillées et notamment le Languedoc-Roussillon. Ainsi, le rééquilibrage géographique des médecins vers les régions moins densément peuplées se fait principalement au détriment des régions «à l'équilibre ». Le niveau des primes est en outre insuffisant pour attirer massivement les généralistes en Île-de-France, et ce, quelle que soit leur région d'origine. En effet, la perte de qualité de vie à exercer dans cette région est très élevée, et le différentiel de revenu est énorme : la plupart des médecins verraient leur revenu diminuer s'ils s'installaient dans cette région. La prime à instaurer par l'état et/ou les collectivités devrait être d'un montant très élevé, supérieur à 10000 euros, pour y attirer suffisamment de médecins.

\section{Une faible influence des caractéristiques individuelles des médecins}

Les seules variables définies au niveau individuel sont les caractéristiques sociodémographiques des médecins (âge à l'installation et sexe). Les résultats des régressions (non présentés ici) montrent que les femmes sont plus à même de s'installer dans les régions PACA, Pays de la Loire, Languedoc-Roussillon, MidiPyrénées et Rhône-Alpes que dans la région Picardie (la région de référence). Les régions du Sud de la France seraient donc relativement plus attractives pour les femmes. Nous l'avions déjà mis en évidence (cf. tableau 1) : la proportion de femmes exerçant dans les régions à forte densité est significativement supérieure à celle exerçant dans les régions à plus faible densité. Ainsi, la région PACA compte $28 \%$ de femmes, le Languedoc-Roussillon en compte $30 \%$; mais elles ne représentent que $19 \%$ dans le Centre ou la Picardie.

Étant donnés ces résultats, nous avons effectué les régressions précédentes séparément pour les hommes et les femmes. Les variables explicatives économiques (telles que le revenu) ainsi que les aménités (soleil, littoral, etc.) influencent identiquement les comportements des hommes et des femmes médecins.

L'âge à l'installation n'a, lui, aucune influence sur le choix de la région d'exercice : cette variable n'est jamais significative ${ }^{12}$. Les médecins qui

12. Cette variable n'est pas introduite sous forme continue, mais en tranche: âge à l'installation inférieur à 31 ans (1 ${ }^{\text {er }}$ quartile), entre 31 et 38 ans, supérieur à 38 ans ( $3^{\text {ème }}$ quartile). 
s'installent plus jeunes ne se tournent pas vers des régions caractérisées par un dynamisme économique et démographique plus important.

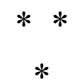

L'analyse économétrique des choix de localisation des médecins généralistes montre que différents outils pourraient être utilisés afin de réguler la répartition des médecins sur le territoire. Les politiques devraient être orientées en premier lieu vers les étudiants en médecine, via des modulations régionales du numerus clausus ou du nombre de places offertes aux épreuves classantes nationales par exemple. Toutefois, ces mesures sont nécessairement contraintes par les capacités de formation des différentes régions. En outre, leur efficacité est limitée par les différences d'attractivité des régions françaises, sur lesquelles le régulateur ne peut agir. Les récentes politiques d'incitations financières à l'installation dans des zones sous-dotées en médecins apparaissent par ailleurs susceptibles d'influencer la localisation des médecins sur le territoire. Toutefois, la modification de la répartition qui en résulte est quantitativement d'une faible ampleur : les incitations ne semblent pas suffisantes pour contraindre les médecins ayant choisi de s'installer dans les régions très ensoleillées du Sud de la France, à s'installer dans d'autres régions initialement moins attractives. Les mécanismes financiers incitatifs ne peuvent donc pas être les seuls leviers de correction des inégalités de répartition des médecins.

Dès lors, il importerait de les combiner à d'autres types de mesures. Il est très probable que des politiques imposant des freins à l'installation dans les zones déjà trop fortement dotées se heurtent à l'hostilité des médecins, profondément attachés à la défense de leur identité libérale. Pourtant, d'autres professions de santé les ont acceptées, et ce depuis plusieurs années déjà. Ainsi, une première piste pourrait consister à contraindre plus fortement la liberté d'installation des médecins par le biais d'un numerus clausus régional au moment de l'installation, comme pour les pharmacies. Une seconde piste serait de n'accepter le conventionnement des jeunes médecins en zone surdotée que dans le cas où ils y remplacent un médecin partant à la retraite, comme pour les infirmières libérales.

Bien que cette étude apporte des résultats originaux, elle comporte toutefois un certain nombre de limites, inhérentes aux données que nous utilisons. Tout d'abord, nos données ne comportent aucune information d'ordre personnel (présence d'un conjoint ou d'enfant, âge des enfants, profession du conjoint, etc.). Or ces facteurs influencent très probablement le choix de la localisation géographique d'un médecin.

Par ailleurs, les données sont relativement anciennes. Couvrant uniquement la période 1997-2002, elles ne permettent pas d'analyser l'impact de plusieurs réformes, postérieures à 2002, qui ont pu influencer les comportements de localisation des médecins installés récemment. Ainsi, nous ne pouvons pas évaluer ex-post l'impact de l'introduction, dès 2004, de mesures financières incitatives à la localisation dans des zones sous-dotées en médecins. Nous ne pouvons pas non plus évaluer les conséquences de la mise en place du parcours de soins coordonnés, en 2004, sur leurs comportements. Fin 2008, 85 \% des assurés avaient désigné un médecin traitant, médecin généraliste dans 99,5\% des cas (CNAMTS, 2009). Dans ces conditions, il se peut que les médecins débutants soient plus réticents à s'installer dans des zones déjà très densément peuplées en médecins, dans lesquelles les patients n'ayant pas encore désigné de médecin traitant sont peu nombreux. Enfin, la réforme des études médicales de 2004 et l'introduction des épreuves nationales classantes, qui conduit les médecins à choisir, en fonction de leur rang de classement, une spécialité mais également un lieu de formation, aura probablement des conséquences importantes sur les comportements d'installation des médecins. Des données plus récentes permettraient d'apporter un nouvel éclairage sur ce sujet. 


\section{BIBLIOGRAPHIE}

Baudier F., Bourgueil Y., Evrard I., Gautier A., Le Fur P. et Mousquès J. (2010), « La dynamique de regroupement des médecins généralistes libéraux de 1998 à $2009 »$, Questions d'économie de la santé, $\mathrm{n}^{\circ} 157$, IRDES.

Ben-Akiva M. et Lerman SR. (1985), Discrete choice analysis, MIT Press.

Bessière S., Breuil-Genier $P$. et Darriné S. (2004), «La démographie médicale à l'horizon 2025 : une régionalisation des projections ", Études et Résultats, n ${ }^{\circ} 353$, DREES.

Bilodeau H. et Leduc N. (2003), « Recension des principaux facteurs d'attraction, d'installation et de maintien des médecins en régions éloignées », Cahiers de Sociologie et de Démographie Médicale, vol. 43, $\mathrm{n}^{\circ}$ 3, pp. 485-504.

Bolduc D., Fortin B. et Fournier M.-A. (1996), « The Effect of Incentive Policies on the Practice Location of Doctors: A Multinomial Probit Analysis », Journal of Labor Economics, vol. 14, $\mathrm{n}^{\circ} 4$, pp. 703-732.

Bolduc D., Lacroix G. et Muller C. (1997), " The Choice of medical providers in rural Benin: a comparison of discrete choice models », Journal of Health Economics, vol. 15, $\mathrm{n}^{\circ} 4$, pp. 477-498.

Bolduc D., Fortin B. et Gordon S. (1997), «Multinomial Probit Estimation of Spatially Interdependent Choice: An Empirical Comparison of Two New Techniques », International Regional Science Review, vol. 20, pp. 77-101.

Bourgueil Y., Mousquès J., Marek A. et Tajahmadi A. (2007a), « Améliorer la répartition géographique des médecins : les mesures adoptées en France ». Questions d'économie de la santé, $\mathrm{n}^{\circ} 122$, IRDES.

Bourgueil Y., Mousquès J. et Tajahmadi A. (2007b), «Comment améliorer la répartition géographique des professionnels de santé ? Les enseignements de la littérature internationale et des mesures adoptées en France ». Document de travail, $\mathrm{n}^{\circ} 1635$, IRDES.

Bui D. et Levy D. (2000). « Les jeunes médecins et l'installation en pratique libérale ». Cahiers de Sociologie et de Démographie Médicale, vol. 40, $n^{\circ} 3-4$, pp. 252-345.
Cameron AC. et Trivedi PK. (2005), Microeconomics: Theory and Applications, Cambridge University Press, New York.

Cereq (2003), « D'une région à l'autre, la mobilité des jeunes en début de vie active », Bref, $\mathrm{n}^{\circ} 198$, Cereq.

CNOM (2008), Atlas de la démographie médicale en France - situation au $1^{\text {er }}$ janvier 2008.

Coreia I. etVeiga P. (2009), « Geographic distribution of physicians in Portugal », European Journal of Health Economics, vol. 11, $\mathrm{n}^{\circ} 4$, pp. 383-393.

Couffinhal A., Lucas-Gabrielli V. et Mousques J. (2002), « Où s'installent les nouveaux omnipraticiens? Une étude cantonale sur 1998-2001 », Questions d'économie de la santé, $\mathrm{n}^{\circ} 61$, IRDES.

Cour des Comptes (2007), «Sécurité Sociale $2007 »$, chapitre 8 («Les médecins libéraux : démographie, revenus et parcours de soins »).

Delattre E. et Dormont B. (2003), « Fixed Fees and Physician-Induced Demand: a Panel Data Study on French Physicians ", Health Economics, vol. $12, \mathrm{n}^{\circ} 9$, pp. 741-754.

Delattre E. et Dormont B. (2005), « La régulation de la médecine ambulatoire en France: quel effet sur le comportement des médecins libéraux ? », Solidarité Santé, n 1, pp. 135-161, DREES.

Dionne G., Langlois A. et Lemire N. (1987), "More on the geographic distribution of physicians », Journal of Health Economics, vol. 6, $\mathrm{n}^{\circ} 4$, pp. 365-374.

Dormont B. et Samson AL. (2009), « Démographie médicale et carrières des médecins généralistes : les inégalités entre générations », Économie et Statistiques, ${ }^{\circ} 414$, pp. 3-30.

Ecosante (2012). http ://www.ecosante.fr/.

Goddard M., Gravelle H, Hole A. et Marini G. (2010), «Where did all the GPs go ? Increasing supply and geographical equity in England and Scotland », Journal of Health Services Research and Policy, vol. 15, $\mathrm{n}^{\circ} 1$, pp. 28-35. 
Gravelle H. et Sutton M. (2001), « Inequalities in the geographical distribution of general practitioners in England and Wales 1974-1995 », Journal of Health Services Research and Policy, vol. $6, \mathrm{n}^{\circ} 1$, pp. 6-13.

Horev T., Psis-Katz I. et Mukamel D. (2004), « Trends in geographic disparities in allocation of health care resource in the US », Health Policy, vol. $68, \mathrm{n}^{\circ} 2$, pp. 223-232.

OCDE (2009), Health at a Glance 2009: OECD Indicators, OCDE.

Raynaud D. (2005), «Les déterminants individuels des dépenses de santé ». Études et résultats, $n^{\circ} 378$, DREES.
Roback J. (1982), « Wages, rents and the quality of life », Journal of Political Economy, vol 90, $\mathrm{n}^{\circ} 6$, pp. 1257-1278.

Simoens S. et Hurst J. (2006), « The supply of physician services in OECD countries », OECD Health Working Papers, $\mathrm{n}^{\circ} 21$, OECD.

Thomas A. (2000), Économétrie des variables qualitatives, Dunod, Paris.

Train K. (2003), Discrete Choice Methods with Simulation, Cambridge University Press.

Vilain A. et Niel, X. (1999), «Les inégalités régionales de densité médicale : le rôle de la mobilité des jeunes médecins ». Études et résultats, $\mathrm{n}^{\circ} 30$, DREES. 


\section{Cadre d'analyse : les modèles de choix discrets}

Pour étudier le choix de la région d'exercice des généralistes, nous utilisons des méthodes économétriques de choix discrets (Ben Akiva et Lerman, 1985), basées sur l'axiomatique de la maximisation de l'utilité. Nous supposons que l'utilité $U_{i j t}$ d'un médecin $i(i=1, \ldots, N)$ à s'installer dans la région $j(j=1, \ldots J)$ à la date $t(t=1, \ldots, T)$ dépend d'attributs spécifiques à l'individu (notés $Z_{j}$ ), d'attributs spécifiques à la région - ou alternative - (notés $X_{j t}$ ) et d'une composante inobservable $\left(\varepsilon_{i j t}\right)$. On a :

$$
\begin{aligned}
U_{i j t}= & X_{j t}^{\prime} \beta+Z_{i}^{\prime} \gamma_{j}+\varepsilon_{i j t} \\
& (i=1, \ldots, N ; j=1, \ldots, J ; t=1, \ldots, T)
\end{aligned}
$$

Le médecin $i$ choisit de s'installer dans la région $j$ à la date $t$ si l'utilité qu'il retire à s'installer dans cette région est supérieure à celle qu'il retirerait à s'installer dans toutes les autres régions, soit :

$U_{i j t} \geq U_{i k t}, \forall k \neq j$

Ainsi, pour chaque médecin $i$, on observe les $\mathrm{J}$ variables binaires $y_{i j t}$, correspondant aux $\mathrm{J}$ alternatives (ou régions) qui s'offrent à lui à la date $t$, et définies ainsi :

$\left\{\begin{array}{l}y_{i j t}=1 \text { si } U_{i j t} \geq U_{i k t}, \forall k \neq j \\ y_{i j t}=0 \text { sinon }\end{array}\right.$

\section{Le modèle Logit conditionnel}

Nous estimons ici un modèle Logit conditionnel. Les erreurs $\varepsilon_{i j t}$ de l'équation (1) sont indépendamment et identiquement distribuées (i.i.d.) et suivent une loi de Gumbel. En outre, elles sont supposées indépendantes des caractéristiques régionales $X_{j t}$ et des caractéristiques individuelles $Z_{i}$.

Dans ce cadre, on obtient une expression explicite de la probabilité de choix de chacune des J régions. La probabilité, pour un individu $i$, de choisir la région $j$ à la date $t$ est notée :

$$
\begin{aligned}
& \operatorname{Pr}\left(y_{i j t}=1\right)=p_{i j t}=\frac{\exp \left(X_{j t}^{\prime} \beta+Z_{i}^{\prime} \gamma_{j}\right)}{\sum_{j=1}^{J} \exp \left(X_{j t}^{\prime} \beta+Z_{i}^{\prime} \gamma_{j}\right)}, \\
& i=1, \ldots, N ; j=1, \ldots, J ; t=1, \ldots, T
\end{aligned}
$$

- Le vecteur des $\mathrm{K}$ variables explicatives $X_{j t}$ $\left(X_{j t}=\left(X_{j t(1)}, X_{j t(2)}, \ldots, X_{j t(K)}\right)\right)$ est constitué de variables spécifiques à la région $j$, i.e. dont la valeur dépend de la région considérée (par exemple, le revenu des médecins observé en moyenne dans chaque région). Ces variables peuvent être constantes, ou non, dans la dimension temporelle. En effet, des médecins qui s'installent dans la même région mais à des années différentes, font face à des caractéristiques régionales différentes.

- Le vecteur des $M+1$ variables explicatives $Z_{i} \quad\left(Z_{i}=\left(1, Z_{i(1)}, Z_{i(2)}, \ldots, Z_{i(M)}\right)\right) \quad$ inclut des variables spécifiques à l'individu $i$, qui sont constantes dans le temps et quelle que soit la région choisie (c'est le cas du sexe par exemple). La première composante du vecteur vaut 1 : notre spécification contient donc des constantes spécifiques à chaque région. Ces constantes reflètent l'attractivité de chaque région, car elles incluent l'ensemble des variables inobservées qui influencent le choix d'une région et qui sont stables dans le temps.

Étant donnée la distribution retenue pour les perturbations $\varepsilon_{i j t}$ et $\varepsilon_{i j^{\prime} t}$, nous faisons l'hypothèse que les caractéristiques inobservables qui expliquent le choix de la région $j$ par les individus ne sont pas corrélées aux caractéristiques inobservables qui expliquent le choix de la région $j$ '. Cette hypothèse d'indépendance des alternatives non pertinentes est relativement forte, notamment si on considère deux régions proches géographiquement. Ainsi, les caractéristiques inobservables qui expliquent le choix de s'installer en région PACA (forte valorisation du climat par exemple) sont probablement corrélées, pour partie, à celles qui expliquent le choix de s'installer en Languedoc-Roussillon. Pour lever cette 
hypothèse, il serait envisageable d'autoriser une corrélation spatiale entre les perturbations de deux régions $j$ et $j$ ' (comme le font Bolduc, et al., 1996) ou encore d'estimer un modèle Probit multinomial, qui n'impose pas de structure particulière sur la corrélation entre les perturbations des différentes régions. Néanmoins, ces modèles sont lourds à mettre en œuvre dans le cas où le nombre d'alternatives est élevé, comme c'est le cas dans cette étude (22 alternatives). Selon Thomas (2000), le modèle Probit multinomial est bien adapté lorsque le nombre de modalités ne dépasse pas quatre.

\section{Interprétation des coefficients}

Dans les modèles Logit conditionnels, seul le signe des coefficients est directement interprétable.

L'écriture (2) montre que, aux variables spécifiques à la région $X_{j t}$ est associé un vecteur constant $(\beta)$ de paramètres à estimer. Ainsi, $\hat{\beta}_{1}>0$ signifie qu'une augmentation de la variable $X_{j t(1)}$ dans une région conduit à une augmentation de la probabilité de choix de cette région au détriment des autres.

Aux variables spécifiques à l'individu $Z_{i}$ est associé un vecteur de paramètres à estimer $\left(\gamma_{j}\right)$ qui dépend de la région. Chaque coefficient estimé est donc interprété par rapport à une région de référence. Un coefficient estimé $\hat{\gamma}_{(1) j}>0$ signifie qu'une hausse de la variable $Z_{i(1)}$ conduit à une augmentation de la probabilité de choisir la région $j$ plutôt que la région de référence.

\section{Calcul des effets marginaux}

Puisque la valeur des coefficients estimés n'est pas directement interprétable, nous calculons les effets marginaux afin de quantifier l'impact des différentes variables explicatives sur la probabilité de choix d'une région. Prenons l'exemple $\mathrm{du}$ revenu, qui est introduit sous forme quadratique. Dans le cadre du modèle (1), l'utilité individuelle dépend du revenu (rev) et d'un ensemble d'autres variables $(X$ et $Z)$. On a :

$$
\begin{gathered}
U_{i j t}=\beta_{1} * \operatorname{rev}_{j t}+\beta_{2} * \operatorname{rev}_{j t}^{2}+X_{j t}^{\prime} \beta+X_{i}^{\prime} \gamma_{j}+\varepsilon_{i j t} \\
(i=1, \ldots, N ; j=1, \ldots, J ; t=1, \ldots, T)
\end{gathered}
$$

L'effet marginal du revenu mesure l'impact d'une hausse de 1 euro de revenus dans la région $j$ à la date $t$ sur la probabilité de choisir cette région. Il se calcule de la façon suivante (Train, 2003) :

$$
\frac{\partial p_{i j t}}{\partial r e v_{j t}}=p_{i j t}\left(1-p_{i j t}\right)\left(\beta_{1}+2 \beta_{2} * r e v_{j t}\right)
$$

Puisque le revenu est introduit sous forme quadratique, l'effet marginal dépend également de la valeur du revenu de la région $j$ à la date $t$. Plutôt que de calculer un effet marginal au point moyen de l'échantillon, nous calculons ici l'effet marginal pour chaque individu puis la moyenne de ces effets marginaux sur l'ensemble des médecins. C'est l'approche qui est généralement utilisée (Cameron et Trivedi, 2005).

\section{Calcul du taux marginal de substitution (TMS) entre revenus et ensoleillement}

Pour calculer le TMS entre revenu et ensoleillement, c'est-à-dire le montant du revenu additionnel nécessaire pour compenser la perte d'une unité d'ensoleillement et maintenir une utilité constante, on se replace dans le cadre de l'équation (1). L'utilité individuelle dépend du nombre d'heures d'ensoleillement (so), du revenu (rev) et d'un ensemble d'autres variables $(X$ et $Z)$. On a :

$$
\begin{aligned}
& U_{i j t}=\beta_{1} * r e v_{j t}+\beta_{2} * r e v_{j t}^{2}+\beta_{3} * s o_{j} \\
&+X_{j t}^{\prime} \beta+X_{i}^{\prime} \gamma_{j}+\varepsilon_{i j t} \\
&(i=1, \ldots, N ; j=1, \ldots, J ; t=1, \ldots, T)
\end{aligned}
$$

Le TMS du revenu au soleil est donc :

$$
T M S=\frac{d U / d s o}{d U / d r e v}=\frac{\beta_{3}}{\beta_{1}+2 * \beta_{2} * r^{2} v_{j t}}
$$

Puisque le revenu est introduit sous forme quadratique dans l'expression de l'utilité, la valeur du TMS dépend du niveau de revenu de la région $j$ à la date $t$ considérée. Le TMS par région est donc calculé en utilisant le niveau moyen de revenu de chaque région.

\section{Simulations : l'effet d'une politique financière incitative sur les comportements de localisation}

Nous simulons les effets d'une politique financière $\mathrm{d}$ 'incitation à la localisation dans les zones sous dotées en médecins sur les comportements de localisation des médecins qui choisissent de changer de région à l'issue de leur thèse. 
Pour cela, nous estimons tout d'abord un modèle dans lequel l'effet du revenu sur les choix de localisation des médecins diffère en fonction du type de la région (région à faible densité $(j \in F)$, à densité moyenne $(j \in M)$ ou à forte densité $(j \in H))$. L'utilité définie dans le cadre du modèle (1) peut se réécrire :

$$
\begin{aligned}
& U_{i j t}=a_{F} * r e v_{j t, j \in F}+b_{F} * r e v_{j t, j \in F}^{2} \\
& +a_{M} * r e v_{j t, j \in M}+b_{M} * r e v_{j t, j \in M}^{2} \\
& +a_{H} * r e v_{j t, j \in H}+b_{H} * r e v_{j t, j \in H}^{2} \\
& +X_{j t}^{\prime} \beta+Z_{i}^{\prime} \gamma_{j}+\varepsilon_{i j t} \\
& (i=1, \ldots N ; j=1, \ldots, J ; t=1, \ldots, T)
\end{aligned}
$$

Nous calculons pour chaque individu $i$ sa probabilité $p_{i j t}$ de s'installer dans la région $j$ à la date $t$, telle qu'elle est décrite par l'équation (2).

La simulation consiste à attribuer 5000 euros ou 10000 euros de revenu supplémentaire aux médecins qui choisissent d'exercer dans les régions dans lesquelles la densité médicale est faible (i.e. pour $j \in F$ ). Après ajout de cette prime à l'installation, nous calculons la nouvelle probabilité, pour chaque médecin $i$, de $\mathrm{s}$ 'installer dans la région $j$ à la date $t$ et obtenons alors la répartition simulée des médecins après mise en place d'une politique de prime à l'installation. Celle-ci est comparée à la répartition initiale des médecins. 